\title{
GmFULa Improves Soybean Yield by Enhancing Carbon Assimilation without Altering Flowering Time or Maturity
}

\section{Yanlei Yue}

Henan Agricultural University

\section{Shi Sun}

Chinese Academy of Agricultural Sciences Institute of Crop Sciences

Jiawen Li

Henan Agricultural University

Haidong Yu

Henan Agricultural University

\section{Hongxia Wu}

Henan Agricultural University

\section{Baiquan Sun}

Chinese Academy of Agricultural Sciences Institute of Crop Sciences

\section{Tao Li}

Henan Agricultural University

\section{Tianfu Han}

Chinese Academy of Agricultural Sciences Institute of Crop Sciences

Bingjun Jiang ( $\square$ jiangbingjun@caas.cn )

Chinese Academy of Agricultural Sciences Institute of Crop Sciences https://orcid.org/0000-00028172-4646

\section{Research Article}

Keywords: soybean (Glycine max (L.) Merr.), GmFULa, yield, biomass, palisade tissue, sucrose synthesis and transport

Posted Date: May 6th, 2021

DOI: https://doi.org/10.21203/rs.3.rs-483237/v1

License: () (i) This work is licensed under a Creative Commons Attribution 4.0 International License. Read Full License 
Version of Record: A version of this preprint was published at Plant Cell Reports on July 16th, 2021. See the published version at https://doi.org/10.1007/s00299-021-02752-y. 
3 GmFULa Improves Soybean Yield by Enhancing Carbon

\section{Assimilation without Altering Flowering Time or}

\section{Maturity}

7 Yanlei Yue ${ }^{1}$, Shi Sun ${ }^{2}$, Jiawen $\mathrm{Li}^{1}$, Haidong $\mathrm{Yu}^{1}$, Hongxia $\mathrm{Wu}^{1}$, Baiquan Sun ${ }^{2}$, Tao $\mathrm{Li}^{1,{ }^{*}}$,

8 Tianfu $\mathrm{Han}^{2, *}$ and Bingjun Jiang ${ }^{2, *}$

$9{ }^{1}$ College of Life Sciences, Henan Agricultural University, Zhengzhou 450002, China

$10{ }^{2}$ MARA Key Lab of Soybean Biology (Beijing), Institute of Crop Sciences, The Chinese

11 Academy of Agricultural Sciences, Beijing 100081, China

12

$13{ }^{*}$ Correspondence (Tel 86-10-82108589; fax 86-10-82108784; email

14 jiangbingjun@caas.cn [B.J.]); (Tel 86-10-82105875; fax 86-10-82108784; email

15 hantianfu@caas.cn [T.H.]); and (Tel 86-371-63555790; fax 86-371-63555790; email

16 litao0504@henau.edu.cn [T.L.]) 


\section{Abstract}

Key message GmFULa improved soybean yield by enhancing carbon assimilation. Meanwhile, different from known yield-related genes, it did not alter flowering time or maturity.

Abstract Soybean is highly demanded by a continuously growing human population. However, increasing soybean yield is a major challenge. FRUITFULL (FUL), a MADS-box transcription factor, plays important roles in multiple developmental processes, especially fruit and pod development, which are crucial for soybean yield formation. However, the functions of its homologs in soybean are not clear. Here, through haplotypes analysis, we found that haplotypes $\mathrm{HO2}$ of the soybean homolog GmFULa (GmFULa-HO2) is dominant in cultivated soybeans, suggesting that GmFULaHO2 was highly selected during domestication and varietal improvement of soybean. Interestingly, transgenic overexpression of GmFULa enhanced vegetative growth with more biomass accumulated and ultimately increased the yield but without affecting the plant height or changing the flowering time and maturity, indicating that it enhances the efficiency of dry matter accumulation. It also promoted the yield factors like branch number, pod number and 100-seed weight, which ultimately increased the yield. It increased the palisade tissue cell number and the chlorophyll content to promote photosynthesis and increase the soluble sugar content in leaves and fresh seeds. Furthermore, GmFULa were found to be sublocalized in the nucleus and positively regulate sucrose synthases (SUSS) and sucrose transporters (SUTS) by 
40 showed GmFULa promotes the capacity of assimilation and the transport of the

41 resultant assimilates to increase yield, and provided insights into the link between

42 GmFULa and sucrose synthesis with transport related molecular pathways that

43 control seed yield.

44 Keywords: soybean (Glycine max (L.) Merr.), GmFULa, yield, biomass, palisade

45 tissue, sucrose synthesis and transport

46

47 Abbreviations

48 CDS coding sequence

49 DAG day after germination

50 FUL FRUITFULL

51 SUS sucrose synthase

52 SUT sucrose transporters

53 ZGDD Zigongdongdou

54 


\section{Introduction}

56 Soybean (Glycine max (L.) Merr.) provides large amounts of edible oils and vegetable

57 proteins for humans and livestock, and thus demand for soybean is increasing 58 globally due to human population growth. However, available cultivated land

59 resources are largely limited, meaning that increasing soybean unit yield, as a way to

60 increase the total yield, is a major challenge. Soybean yield is based on pod number

61 per plant, seed number per pod and 100-seed weight (Roekel et al. 2015; Yan et al.

62 2017; Bianchi et al. 2020). Taking into account that soybean is sensitive to

63 photoperiod, maturity loci have important roles in yield. However, there are many

64 yet unknown quantitative loci reported to be linked to yield-related traits in SoyBase

65 (https://www.soybase.org/) but only a few loci have been molecularly identified.

66 Among them, GmCYP78A10 is related to pod number and 100 -seed weight and $L n$ is

67 a major gene controlling four-seed pod development in soybean (Jeong et al. 2012;

68 Wang et al. 2015; Sayama et al. 2017). GsCID1 is responsible for 100-seed weight in

69 wild soybean ( $\mathrm{Hu}$ et al. 2020). Recently, GmKIX8-1 was demonstrated to be

70 associated with soybean seed weight (Nguyen et al. 2021). However, the molecular

71 mechanism controlling yield in soybean is largely unknown.

72 FRUITFULL (FUL), a MADS box transcription factor, has essential and pleiotropic roles

73 in multiple developmental processes including shoot initiation, reproductive

74 transition, inflorescence differentiation and fruit development (Ferrandiz et al. 2000;

75 Balanza et al. 2019; Maheepala et al. 2019; Zhang et al. 2019a; Zhao et al. 2019). In 
76 Arabidopsis, FUL (AGL8) is the main member of the regulatory network that

77 determines fruit growth pattern (Mandel Yanofsky 1995; Alvarez-Buylla et al. 2019). FUL down-regulates APETALA2 and INDEHISCENT, and they two promote pod elongation (Mandel Yanofsky 1995; Balanza et al. 2019; Di Marzo et al. 2020). FUL is necessary for terminal flower formation in seedless states (Balanza et al. 2019). FUL also contributes to the differentiation of inflorescence, stem, leaf and carpel (Zhang et al. 2013; Yao et al. 2019). Similar functions were found in the pea and cotton homologous genes VEGETATIVE1 and GhMADS22, respectively (Berbel et al. 2012; Zhang et al. 2013). CSFUL1 regulates fruit length in cucumber, and DEFH28, a homologous gene in Antirrhinum japonicum, regulates carpel wall differentiation and fruit ripening (Müller et al. 2001; Zhao et al. 2019). In tomato, TDR4/FUL1 and MBP7/FUL2 are involved in cell wall modification and affect fruit ripening without dependence on ethylene (Bemer et al. 2012; Li et al. 2019). FUL can also modulate plant architecture. Rice homolog OsMADS18 is negatively related to the number of tillers, and controls the branch angle by inhibiting SAUR10 (SMALL AUXIN UPREGULATED RNA 10) expression (Bemer et al. 2017).

In addition, FUL functions in the formation of secondary metabolites. VmTDR4 is involved in the accumulation of anthocyanins during the normal ripening period in blueberries, and DR4/FUL1 and MBP7/FUL2 participate in the synthesis of volatile substances in tomato fruits (Jaakola et al. 2010; Bemer et al. 2012) and affect the accumulation of pigment in tomato maturity (Wang et al. 2014). Moreover, in Arabidopsis, FUL can intertalk with hormone- and light-signaling pathways. It directly 
111 Here, we analyzed the molecular function of GmFULa. First, we found that it has six

112 haplotypes with GmFULa-H01 and GmFULa-H02 dominant. GmFULa-H01 is dominant

113 in wild soybeans, while GmFULa-HO2 is dominant in cultivated soybeans especially

114 southern cultivars, indicating that it is an elite allele for soybean breeding.

115 Overexpression of GmFULa-HO2 results in more biomass and higher yield with

116 increased seed number and weight. We further show that whole-soluble sugar

117 contents were increased in fresh leaves and seeds. Consistently, GmFULa bound to

118 the promoters of GmSUS12 and GmSUT5 and activated their expression. The cell

119 distribution in palisade tissue regulated by GmFULa and chlorophyll content 
120 increased significantly with high photosynthetic efficiency in an overexpression line.

121 Our results show that the GmFULa-GmSUSs/GmSUTs pathway regulates the seed

122 yield of soybean, controls the cell number of palisade tissue, and enhances organic

123 matter accumulation by increasing whole-soluble sugar contents in leaf tissue. In

124 contrast, it doesn't affect flowering time or maturity.

125 Materials and methods

126 Cloning and sequence analysis of GmFULa

127 The sequences of GmFUL family genes and their encoding proteins were obtained

128 from Phytozome (https://phytozome.jgi.doe.gov). Conserved domains were

129 searched in the NCBI database. The CDS of GmFULa was amplified from the soybean

130 cultivar Zigongdongdou (ZGDD) with the primers listed in Supplementary Table S1.

131 Then the PCR products were cloned into the pZeroBack/blunt vector (TianGen,

132 Beijing, China). Sanger sequencing was then performed to confirm the sequences

133 and variations (Shanghai Sangon Biological Engineering Technology and Service CO.,

134 LTD, Zhengzhou, China).

135 Haplotype analysis of GmFULa

136 Publicly available genome resequencing data (NCBI: SRP062560, SRP045129) was

137 used. These sequences were mapped to the Williams 82 genome (v275) using bwa

138 v0.7.10 with default parameters. SNPs/indels were called using the

139 UnifiedGenotyper module (-stand_call_conf 30.0 -stand_emit_conf 10.0) of the

140 GenomeAnalysisTK suite (https://gatk.broadinstitute.org/) (Zhang et al. 2019b). The 
141 polymorphism information of GmFULa was further extracted. The SNP/InDel sites

142 located in the CDS region and including missense mutations were selected to define

143 haplotypes and perform haplotype analysis (Jiang et al. 2019).

Plant materials and growth conditions

145 ZGDD is a photoperiod-sensitive cultivar suitable of low latitude conditions. In this

146 study, ZGDD was used as a wild type control for molecular analysis including genetic

147 transformation. To investigate soluble sugar content, chlorophyll content, sucrose synthase activity, biomass, cell morphology and gene expression, plants were grown

149 in pots containing a 1:1 mixture of forest vermiculite in a light chamber (20000 Lux)

150 under short-day condition $(12 \mathrm{~h} / 12 \mathrm{~h})$ at $25^{\circ} \mathrm{C}$ with $60 \%$ humidity.

151 Plants for the investigation of maturity, yield related traits and photosynthetic

152 activity were grown under field conditions (with $1.5 \mathrm{~m}$ row length, $75 \mathrm{~cm}$ row spacing, $10 \mathrm{~cm}$ plant spacing, three replicates and completely randomized design) from November of 2016, 2019 and 2020 to next Aprils in tropical city of Sanya $\left(18.1^{\circ} \mathrm{N}, 109.2^{\circ} \mathrm{E}\right.$, mean temperature $\left.26^{\circ} \mathrm{C}\right)$, Hainan province, China.

\section{Creation of transgenic overexpression plants of GmFULa}

For the GmFULa over-expression construct, the full-length coding sequence of GmFULa from ZGDD was cloned into the binary vector pTF101.1 between the Xbal and Sacl sites, downstream of the constitutive Cauliflower Mosaic Virus 35S promoter (Yue et al. 2017). The transformation of soybean followed the method of

161 affecting Agrobacterium-mediated using the cotyledonary node explant (Paz et al. 2004). Transgenic plants were verified by PCR-based markers with primers listed in 
163 Supplementary Table S1. The soybean transgenic lines were advanced to the T5

164 generation.

Soluble sugar determination

166 The middle leaflet of the second fully-expanded trifoliolate leaves in the V2 stage

167 and the seed of R6 stage were used. The relative content of soluble sugar was

168 determined with the Anthrone-sulfuric acid colorimetry method using the soluble

169 sugar extract of ZGDD as reference (Huang et al. 2020).

170 Sucrose synthase activity measurement

171 The sucrose synthase activity was measured with a Sucrose Synthase Activity Assay

172 Kit (boxbio, Beijing, China). The relative sucrose synthase activity was expressed as

173 the ratio between the sucrose synthase activities of transgenic lines and ZGDD.

174 Chlorophyll concentration measurement

175 The middle leaflet of the second fully-expanded trifoliolate leaves in the R2 stage

176 (full blooming) were collected. The chlorophyll was extracted and measured with the

177 method described by Xu et al. (2013).

Biomass measurement

179 The whole plants of 3, 7 and 15 DAG were used and cut into separate shoot and root

180 parts from the cotyledon node. Then the shoot and root samples were sterilized at

$181105^{\circ} \mathrm{C}$ for 30 minutes, dried to constant weight, and weighed.

182 Photosynthesis rate analysis

183 For each group, fifteen plants were randomly selected. The middle leaflet of the

184 second fully-expanded trifoliolate leaves in the V3 stage was used. The 
photosynthesis measurement was conducted with the Li-6400 portable

photosynthesis measuring system (LI-COR, USA) (Singsaas et al. 2004; Xu et al. 2013;

Busch 2018). The photo synthetically active radiation was set up as $1,200 \mu \mathrm{mol}$ photons $\mathrm{m}^{-2} \mathrm{~s}^{-1}$ (Xu et al. 2013).

\section{Leaf morphology and anatomy}

190 The middle leaflet of the third trifoliolate leaf from top was sampled for anatomy

191 analysis, which was performed by Servicebio, China (https://www.servicebio.cn/).

192 The sections were stained with safranin $O$ and fast green (Langdale et al. 1989),

193 mounted with neutral balsam and scanned with a Pannoramic 250 Flash II Scanner

194 (3DHISTECH Kft., Budapest, Hungary). The thicknesses of the leaf, spongy mesophyll,

195 stratum corneum and palisade, the numbers of veins and palisade tissue cells, and

196 the cell sizes of the upper epidermis and palisade cells were evaluated at five points.

197 Gene expression analysis

198 The middle leaflet of the second expansion trifoliolate leaves of soybean V3 stage

199 were sampled. RNA of transgenic plants and wild type ZGDD was isolated using

200 TRIZol (ET111), and reverse-transcribed into cDNA with Easyscript ${ }^{\circledR}$ one-step gDNA

201 removal and cDNA synthesis superMix (AT311) (Transgen Biotech, Beijing, China).

202 The cDNA concentrations were normalized to the GmActin expression levels for 203 quantitative PCR analysis (Quant Studio ${ }^{\mathrm{TM}} 12 \mathrm{~K}$ Flex). The qPCR primers are listed in 204 Supplementary Table S1. The primer specificity and efficiency verification used 205 primer blast in NCBI (https://www.ncbi.nlm.nih.gov/). PCR cycle conditions as hold 206 stage: $95^{\circ} \mathrm{C} 20 \mathrm{~s}$, then PCR stage: $\left(95^{\circ} \mathrm{C} 1 \mathrm{~s}, 60^{\circ} \mathrm{C} 20 \mathrm{~s}\right) \times 50$ cycle, and melt curve stage: 
$20795^{\circ} \mathrm{C} 15 \mathrm{~s}, 60^{\circ} \mathrm{C} 1 \mathrm{~min}, 95^{\circ} \mathrm{C} 15 \mathrm{~s}$. The relative expression levels were estimated using

208 the $2^{-\triangle \Delta C T}$ method (Taylor et al. 2019). Three biological replicates were included.

\section{Transient expression in soybean protoplasts}

210 The GmFULa CDS without the stop codon was amplified and fused to the $5^{\prime}$ end of

211 the open reading frame encoding GFP in pTF101 (Yue et al. 2017), which was driven

212 by the CaMV35S promoter. For the ProGmSUS12YFP construct, a genomic DNA

213 sequence (from $-1,224$ to $-1 \mathrm{bp}$ ) upstream of the GmSUS12 coding sequence was

214 amplified using sequence-specific primers and the sequence was cloned into Kpnl

215 and Xhol sites of the pYFPLT vector, which contains the yellow fluorescent protein

216 (YFP) coding sequences. The recombinant construct was transformed into soybean

217 protoplasts.

218 Selected 14-day soybean leaves were cut into $1 \mathrm{~mm}$ strips and incubated with the

219 enzyme digestion solution ( $1 \%$ Cellulase "Onozuka" Rs, $0.5 \%$ Pectolase Y-23, 9\%

220 Mannitol) in the dark for $5 \mathrm{~h}$ at $25^{\circ} \mathrm{C}$. An equal volume of CPW9M solution was

221 added (Frearson et al. 1973). Digested tissues were filtered through a molecular

222 sieve with 100 mesh number. After centrifugation at $200 \times g$ for $5 \mathrm{~min}$ at $4^{\circ} \mathrm{C}$, the

223 protoplast was washed and precipitated three times with CPW9M. Plasmid $(10 \mu \mathrm{g})$

224 was added to $100 \mu \mathrm{L}$ protoplasts, which were resuspended in MMG and incubated

225 with $110 \mu \mathrm{L}$ PEG4000 solution for $15 \mathrm{~min}$ at $25^{\circ} \mathrm{C}$. After $600 \mu \mathrm{L}$ W5 stop solution was

226 used to end the transfection the protoplasts were washed twice with CPW9M or W5

227 as described previously (Yoo et al. 2007). Finally, protoplasts were cultured in

$228 \mathrm{CPW} 9 \mathrm{M}$ for $20 \mathrm{~h}$ in the dark at $25^{\circ} \mathrm{C}$. Fluorescence images were taken using a 
confocal laser scanning microscope.

230 Protein expression, purification and electrophoretic mobility shift assay (EMSA)

231 For expression of GmFULa protein in bacteria, the GmFULa full-length coding 232 sequences were inserted into the inducible expression vector pET-32a (with $6 \times$ His

233 Tag) between BamHI and Sacl sites. The resulting plasmids were transformed into

234 Escherichia coli strain Rosetta (DE3) and induced using $0.4 \mathrm{mM}$ isopropyl-b-ß-1235 thiogalactopyranoside (IPTG) at $25^{\circ} \mathrm{C}$ for $12 \mathrm{~h}$. The recombinant protein was purified 236 using Proteinlso ${ }^{R}$ Ni-NTA Resin (Transgen biotech, Beijing, China) according to the

237 manufacturer's protocol. For GmSUSs and GmSUTs, the probe fragment consisted of 238 a region of $40 \mathrm{bp}$ with the canonical $\mathrm{CArG}$ box $\left(\mathrm{C}[\mathrm{A} / \mathrm{T}]_{8} \mathrm{G}\right)$ in the center (Table $\mathbf{S 1}$ for 239 primer sequences). The mutated CArG box fragment TM was CCGCG (AATAT) in the 240 mid region of the TG motif. The probes were labeled using the EMSA Probe Biotin

241 Labeling Kit (Beyotime Biotechnology). The same fragments without biotin labeling 242 were used as competitors. The protein-DNA complexes were separated with $6 \%$ 243 native polyacrylamide gels. The Biotin-labeled probes were visualized using 244 Chemiluminescent Biotin-labeled Nucleic Acid Detection Kit (Beyotime 245 Biotechnology) according to the manufacturer's protocol. 


\section{Results}

GmFULa has two highly conserved, dominant haplotypes distributed in both wild and cultivated soybeans

Based on public soybean resequencing data (NCBI:SRP020131, SRP062560, SRP045129 and PRJNA589345) from several whole-genome resequencing studies 252 (Lam et al. 2010; Zhou et al. 2015; Zhang et al. 2019b), we analyzed the 253 polymorphisms of GmFULa in 549 lines including 86 wild soybeans. We found 161 254 variation sites, of which there were 141 SNPs (single nucleotide polymorphisms) and 25520 indels (insertions and deletions), including two synonymous mutation sites (black 256 lines in Figure 1a) and three missense mutation sites in the CDS (coding sequence) 257 region (blue lines in Figure 1a). Based on these five CDS variations, GmFULa was 258 divided into six haplotypes: $\mathrm{H} 01-\mathrm{H} 06$, of which $\mathrm{HO}$ and $\mathrm{HO} 2$ were the most dominant

259 (Figure 1b). Moreover, in wild soybean, H01 was nearly the only haplotype. However, $260 \mathrm{HO1}$ and $\mathrm{HO2}$ were distributed nearly equally in the 80 widely-planted cultivated 261 soybeans from Northeast China, while H02 was dominant in the 54 widely-planted 262 cultivated soybeans from the Huang-Huai-Hai region and Southern China (Figure 1b). 263 These results indicated that $\mathrm{HO2}$ is an elite haplotype related to soybean 264 geographical adaptation. Combined with the observation that GmFULa was highly 265 expressed in the shoot apices (Jia et al. 2015), which strongly indicates that GmFULa 266 should have an important role in yield. However, its actual function is unclear. 
268 Due that GmFULa has three highly conserved homologs in soybean genome, and 269 especially GmFULa is nearly identical to GmFULb with the identity rate as $92.2 \%$ in 270 amino acid sequence and $94.1 \%$ in nucleotide sequence, respectively

271 (Supplementary Figure S1), it is reasonable that GmFULa nullification through 272 CRISPR/Cas9-based gene editing system will be compensated by these homologs 273 especially GmFULb. Thus, to further evaluate the function of GmFULa, we only 274 conducted a conventional overexpression analysis at this time. We cloned GmFULa 275 from soybean variety Zigongdongdou (ZGDD, with the haplotype of GmFULa-HO2) 276 and constructed several transgenic overexpression lines where GmFULa was driven 277 by the CaMV35S promoter. Homozygous transgenic lines were screened and 278 identified by the combination of an herbicide test and PCR identification each 279 generation (Supplementary Figure S2a and b). As expected, the expression levels of 280 GmFULa, examined by quantitative PCR (qPCR), were significantly higher in the 281 transgenic plants (FU64 and FU123, $p<0.01$; FU160, p<0.05) than in the control 282 ZGDD (Figure 1c). However, when determining whether GmFULa promotes plant 283 growth, we found that although the transgenic and control plants reached the 284 vegetative stages VE (emergence) and VC (unifoliolate leaves unrolled) on the same 285 day, days to the subsequent vegetative stages V1 (one trifoliolate leaf unrolled) and 286 V2 (two trifoliolate leaves unrolled) of transgenic lines were respectively about 2 287 days and 5 days shorter than those of the control $(p<0.01)$. In contrast, the days to 288 the reproductive stages R1 (first flowering) and R8 (full maturity) of transgenic lines 
were nearly equal to those of the control (Figure 1d). These results indicated that

290 GmFULa neither affected germination nor maturity but promoted vegetative growth.

\section{GmFULa enhances the accumulation of biomass in a robust way}

292 Consistent with our previous observation, in terms of whole plants, transgenic lines

293 had the same number of nodes and leaves as the control ZGDD at 3 and 7 DAG (days

294 after germination), while they had one more node and trifoliolate leaves at 15 DAG

295 (Figure 2a-f). Wild-type plants reached the vegetative stage of V1 at 15 DAG, while

296 transgenic lines had already entered the next stage of V2 (Figure 2c), indicating that

297 transgenic lines are more vigorous than the wild-type soybean. Moreover, for the

298 three observation time points (3, 7, and 15 DAG), vegetative organs (cotyledons and

299 leaves) were significantly bigger in transgenic soybeans than the wild-type control

300 (Figure 2d-f). Consistently, transgenic lines accumulated significantly higher dry

301 biomass of both shoot and root compared to wild-type plants at 3, 7 and 15 DAG

302 (Figure $\mathbf{2 g}$ ). These results suggested that GmFULa to promotes vegetative biomass

303 accumulation in soybean by increasing the vigor of plants.

\section{GmFULa regulates soybean sink content with increasing soybean yield}

305 Although GmFULa promotes vegetative growth and biomass accumulation leading to

306 increase the source capacity at the vegetative stage, it is necessary to further confirm

307 whether GmFULa can promote the transformation of carbohydrate source-sink to 308 increase soybean yield. To correspond with actual production practices, we evaluated 
309 the yield potentiality of GmFULa under natural field conditions. Transgenic

310 overexpression lines (FU64, FU123, FU160) and wild-type control (the transgenic

311 receptor cultivar ZGDD) were grown in an experimental field in Sanya, Hainan

312 province. Yield-related traits were investigated, including the branch number, plant

313 height, node number, pod number, and seed number as well as overall yield. In

314 keeping with the results of the experiments performed in incubators, the GmFULa-

315 overexpression plants grew better compared to the wild-type control with more pods

316 and more branches but similar height (Figure 3).The seed sizes of transgenic lines

317 were also bigger than the wildtype (Figure 3a). Consistently, the yield-related traits of

318 branch number, node number, pod number, and seed number as well as overall yield

319 all increased significantly $(p<0.01)$ in transgenic lines (Figure $\mathbf{3 b}$ - $\mathbf{g}$ ). However, plant

320 height did not show a significant difference between transgenic lines and wild-type

321 control (Figure 3c). Moreover, FU160 had the highest GmFULa expression level

322 among the three overexpression lines; similarly, it had the highest values of yield-

323 related traits branch number, node number, pod number, and seed number and

324 overall yield. These results indicated that GmFULa increased soybean source content

325 and promoted the source-sink transformation to increase soybean yield. No

326 significant difference was observed in plant height between the overexpression lines

327 and the wild-type control (Figure 3c), suggesting that GmFULa is a candidate gene for

328 ideal plant architecture with shorter node spacing that is more conducive to the

329 utilization, transportation and storage of energy and materials from the source 330 organ. 
332 To understand how GmFULa regulates yield-related traits, we further investigated

333 how leaves, a major source organ, were changed by GmFULa overexpression. Various

334 experiments provided direct hints that GmFULa increases leaf size and thickness, so

335 the potential role of GmFULa in the shape and morphology of leaf cells should be

336 clarified. We performed microscopic observations of leaf transects of a middle leaflet

337 at the same trifoliolate node 25 DAE (days after emergence) of the typical

338 overexpression line FU160 and the wild-type control ZGDD (Figure 4a, b).

339 Consistently, the leaf of the transgenic line was significantly thicker than that of the

340 wild-type control (Figure 4c-e). The numbers of veins and palisade tissue cells in

341 transgenic lines increased significantly compared to those of the wild-type control

342 (Supplementary Table S2 and Figure 4d). The spongy mesophyll, stratum corneum

343 and palisade were thicker in the transgenic line than in the wild-type control (Figure

$344 \mathbf{4 f - h}$ ), though the difference was not significant for spongy mesophyll. In addition, the

345 cell size of the upper epidermis (FU160 $=434 \mu \mathrm{m}^{2}, \mathrm{ZG}=272 \mu \mathrm{m}^{2}$ ) and the thickness of

346 the stratum corneum (FU160 $=5.44 \mu \mathrm{m}, Z \mathrm{ZG}=2.90 \mu \mathrm{m})$ increased significantly in

347 transgenic line FU160 (Figure 4i-j and Supplementary Table S2). Palisade cell size in

348 FU160 was smaller than in control ZGDD (Figure 4k). These data suggest that

349 GmFULa has an important role in regulating cell distribution and palisade

350 development in leaf photosynthesis. 
352 To study the physiological function of GmFULa in soybean growth, we determined

353 chlorophyll content, photosynthesis rate, soluble sugar content and sucrose synthase

354 activity. For chlorophyll content, leaf blades were sampled with a hole punch from

355 the middle leaflet of the second fully-expanded trifoliolate leaves of three plants in

356 the V2 stage and weighed. The content of chlorophyll of overexpression plants were

357 significantly higher than those of the wild-type control (Figure 5a). Correspondingly,

358 the leaf-level photosynthesis rates of transgenic plants were also significantly

359 enhanced in the field conditions (Figure $\mathbf{5 b}$ ). The content of soluble sugar in leaves of

360 V2 stage and seeds of R6 stage were further detected. Compared to WT, transgenic

361 overexpression lines exhibited significantly higher levels of whole-soluble sugar both

362 in leaves and seeds (Figure $\mathbf{6 a}, \mathbf{b}$ ). Consistently, the activity of sucrose synthase of

363 transgenic overexpression lines was higher than that of the wild-type control, though

364 this difference was only significant for FU160 (Figure 6c). These physiological data

365 indicated that GmFULa enhances assimilation in soybean.

\section{GmFULa binds to the conserved CArG boxes present in the promoter regions of}

\section{GmSUS12 and GmSUT5}

To understand how GmFULa regulates agronomic and physiologic traits, we further analyzed its protein subcellular localization. By transient expression of GmFULa-GFP (green fluorescent protein) driven by CaMV35S promoter in soybean protoplasts, we found that the fusion protein was localized in the nucleus of soybean protoplasts based on the observation that the GFP signal was exclusively co-localized with the 
mCherry-labeled nuclear signal (Figure 6d). These results were in line with the

374 prediction that GmFULa should be a transcription factor.

375 Considering that soluble sugar synthesis was promoted in the transgenic lines, it is a 376 reasonable hypothesis that GmSUSs and GmSUTs should be regulated by GmFULa. 377 To clarify this hypothesis, we first performed a qPCR experiment to compare the 378 expression of 12 GmSUSs and eight GmSUTs, and found that most GmSUSs and 379 GmSUTs (especially GmSUS12 and GmSUT5) had significantly higher expression in 380 overexpression lines FU64, FU123 and FU160 leaves of V3 stage than in control plant 381 ZGDD. This result suggests that GmSUS and GmSUT are regulated by GmFULa in 382 soybean growth (Figure 6e).

383 To further confirm whether GmFULa regulates GmSUS and GmSUT directly, 384 GmSUS12 and GmSUT5 were selected for further analysis. In the 2,000 bp upstream 385 promoter region of both GmSUS12 and GmSUT5, we found three (SA-SC) and seven 386 (TA-TG) FUL-combining CArG boxes, respectively (Figure 6f), which indicated that 387 GmFULa might combine these boxes to regulate the expression of GmSUS12 and GmSUT5. Then we performed an EMSA, and found a shift for all detected CArG boxes

389 (SA-SC and TA-TG) as predicted, confirming that GmFULa can physically bind to the 390 promoters of GmSUS12 and GmSUT5 (Figure 6f). Furthermore, a yeast one hybrid 391 experiment also showed that GmFULa binds to GmSUS12 promoter. These results 392 indicate that GmFULa promotes the activity of sucrose synthesis and transport 393 related genes in soybean. 


\section{Discussion}

414 Different from our expectation that GmFULa might regulate soybean maturity and 415 improve soybean adaptation, GmFULa overexpression does not alter the maturity

FRUITFULL (FUL), a MADS-box transcription factor, is essential in the network that regulates the initiation of shoots and buds, the transformation of reproductive growth and the development of organs. In Arabidopsis thaliana, FUL down-regulates AP2 (APETALA2) and IND (INDEHISCENT) and promotes pod elongation (Di Marzo et al. 2020). In cucumber, CSFUL1 regulates fruit length (Zhao et al. 2019). DEFH28 from Antirrhinum majus regulates carpel wall differentiation and fruit ripening (Müller et al. 2001). Bemer et al. (2012) found that TDR4/FUL1 and MBP7/FUL2 affected fruit ripening independent of ethylene. Similarly, our previous study found that GmFULa is specifically expressed in flowers and pods, and is related to photo-thermal adaptation of soybean (Jia et al. 2015). However, we knew less about the exact role of GmFULa on soybean maturity and yield and how it works.

Using publicly available whole genome resequencing data, we found that GmFULa had two major haplotypes, GmFULa-HO1 and GmFULa-HO2. The proportion of GmFULa-HO2 is highest in cultivated soybeans from Middle and South China and second-highest in cultivated soybeans from Northeast China, but nearly absent in wild soybeans. These results indicate that GmFULa-HO2 is an elite allele to be highly selected during domestication and improvement of cultivated soybeans and might promote the expansion of soybeans from high latitudes to low latitudes. 
416 structure, or the durations of vegetative and reproductive growth, which is similar

417 with FUL (AGL8) of Arabidopsis and AaFUL1 of Anthurium (Gu et al. 1998; Ma et al.

418 2019). However, the FUL homologs in Medicago truncatula and Platanus acerifolia

419 promote flowering (Jaudal et al. 2015; Zhang et al. 2019a). In combination with the

420 observation that GmFULa promotes vegetative growth, GmFUL might function in a

421 novel mechanism, as its ancestral homolog Dt2 does in determining semi-

422 determinacy (Liu et al. 2016).

423 GmFULa has an important role in plant architecture through regulating the branch

424 number, node number, leaf size, and leaf thickness. However, GmFULa had no

425 significant effect on plant height when overexpressed. Moreover, it promoted the dry

426 mass accumulation of both root and shoot and ultimately increased yield. In contrast,

427 GmAP1a was found to control flowering time and plant height (Chen et al. 2020).

428 These results indicate that GmFULa has pleiotropic roles on soybean growth and

429 development. GmFULa promotes soybean yield, which increases adaptation. It is

430 significantly different from known maturity loci, which regulate soybean maturity to

431 improve soybean adaptation.

432 GmFULa regulates the source-sink balance. Before reproduction, GmFULa promotes

433 vegetative growth and increases biomass accumulation, as indicated by the

434 observations that chlorophyll content, photosynthesis rate, sucrose synthase activity,

435 soluble sugar content, node number and branch number were all increased in

436 transgenic soybeans compared to the wildtype soybean. However, considering that

437 the number and size of seeds and the final yield were increased in transgenic 
438 soybeans compared to the wildtype soybean, GmFULa effectively promotes the 439 source-sink transition during reproduction. Although the photosynthesis rates were 440 smaller than the ones in Koester et al. (2016), our results were consistent with Lin et 441 al. (2015) for the wildtype soybean Zigongdongdou. It might be possibly due to the 442 genetic background difference: different from the soybeans in Koester et al. (2016) 443 which are all maturity group III cultivars, Zigongdongdou is a MGX cultivar. 444 Moreover, both the chlorophyll content and the photosynthesis rate were improved, 445 which is consistent with the observation that photosynthesis rate is highly correlated 446 with chlorophyll contents (BUTTERY BUZZELL 1977). Considering that the 447 enhancement of leaf-level photosynthesis benefited from chl mutant did not 448 necessarily resulted in canopy-level improvement (Slattery et al. 2016; Slattery et al. 449 2017), it is necessary to further explore the effects of GmFULa on canopy-level 450 processes.

451 More importantly, consistent with our observation that GmFULa regulates the 452 source-sink balance, it also regulates the expression of the sucrose synthase 453 GmSUS12 and the sucrose transporter GmSUT5 through binding to their promoters. 454 SUS is a key enzyme of sucrose metabolism, with an important role in the process of 455 yield formation (Gessler 2021). Overexpressing potato SUS gene can increase cotton 456 yield significantly ( $\mathrm{Xu}$ et al. 2012). Moreover, it is also reported to be related to 457 caryopsis development in rice, nitrogen fixation in legumes and plant response to 458 stresses (Huang et al. 1996; Arrese-lgor et al. 1999; Xiao et al. 2014). SUTs are a kind 459 of typical membrane binding protein, which are widely distributed in various tissues 
460 and organs of higher plants (Barker et al. 2000; Williams et al. 2000). They are 461 responsible for the transmembrane transport of sucrose, and have a significant role 462 in sucrose entering and leaving phloem, sucrose storage and sucrose supply to the 463 sink tissues (Breia et al. 2020; Wang et al. 2020). Combining the increase in soluble 464 sugar in leaves and seeds in transgenic lines, a working model of GmFULa was 465 proposed (Figure 7). In the model, GmFULa regulates the expression of SUS to 466 promote sucrose biosynthesis in the source organ leaf. GmFULa also regulates the 467 expression of SUT to promote sucrose transportation into the sink organ pod. Thus, 468 GmFULa can synchronize both sucrose biosynthesis and transportation to increase 469 the source-sink transition rate of the photosynthesis product and the photosynthesis 470 rate is promoted as a result, which is consistent with the hypothesis proposed by 471 Ainsworth et al. (2004). Moreover, as indicated by the observation that FUL 472 homologs promote pod development, GmFULa efficiently promotes the utilization of 473 sucrose in the sink organ pod to increase the final yield suggesting that GmFULa 474 might also function in pod development. Moreover, GmFULa promotes root 475 development, which means that more nutrients can be absorbed to support 476 development of the above ground parts of plants.

477 GmFULa provides a new option for yield improvement of soybean. Soybean is a short 478 day crop that is sensitive to photoperiod, and the yield is highly dependent on 479 adaption to local photo-thermal environments (Yue et al. 2017). Thus, in the 480 breeding history of soybean, the first trait to address is photoperiod-sensitivity, 481 specifically, modifying maturity to match the soybean photoperiod-sensitivity to local 
environments. Multiple soybean maturity loci have been identified. With the discovery and application of maturity loci, soybean has expanded to higher and lower latitudes, resulting in huge increases in soybean production. If the production increase mainly resulting from maturity adaption can be called the first-generation revolution of soybean breeding, the second-generation revolution of soybean 487 breeding will be the improvement of traits other than maturity. In current breeding programs/strategies, the parental lines with different genetic backgrounds of maturity loci are challenging in conventional hybrid breeding, requiring efforts to find optimal photo-thermal environments for their filial lines through field experiments in 491 different locations. Many known yield loci are highly linked to maturity traits, thus 492 transgenic modification of their causal genes might also have secondary effects that 493 alter the maturity trait, and as a consequence, the main effect of yield will be 494 uncertain if the optimal photo-thermal environments are changed. However, because 495 GmFULa can improve the yield without altering the maturity, it is possible to directly 496 improve elite cultivars without changing maturity. For an elite cultivar, we can modify 497 the expression of GmFULa through transgenic overexpression or through gene498 editing to introduce an enhancer element, remove an inhibitor element or make a 499 haplotype shift, consequently enhancing the yield capacity without significantly 500 changing the optimal ecoregion.

501 In summary, we have functionally characterized GmFULa, a member of the MADS 502 box family in soybean. GmFULa has pleiotropic roles in soybean growth and 503 development. It increases soybean adaptation through promoting vegetative growth 
504 and reproductive growth to increase soybean yield. It promotes source accumulation 505 and the sink transformation, but does not affect maturity. Overexpression of GmFULa 506 thus provides a new way to increase soybean yield and soybean adaptation.

507 
508

509 We thank Mrs Jinlu Tao, Mr Haifeng Hong and Mr Enoch Sapey from the Institute of

510 Crop Sciences, Chinese Academy of Agricultural Sciences for their assistance in

511 soybean planting and management. This work was supported by the National Natural

512 Science Foundation of China (32001573), the National Key R\&D Program of China

513 (2017YFD0101400), the China Agriculture Research System (CARS-04) and the CAAS

514 Agricultural Science and Technology Innovation Project.

\section{Author contributions}

516 Y.Y., B.J., T.H., and T.L. conceived the project; Y.Y, B.J. and S.S. performed the field

517 experiments. Y.Y., T.L., B.J., S.S., J.L., H.W., and B.S. performed the indoor 518 experiments; B.J., and Y.Y. performed the data analysis. Y.Y., B.J., T.H., T.L., and H.Y. 519 wrote the manuscript.

\section{Declarations}

521 Conflict of interest The authors declare no conflict of interests.

522 Ethical approval This article does not contain any studies with human participants or

523 animals performed by any of the authors. 


\title{
Figures
}

\section{Figure 1 Overexpression of GmFULa promotes vegetative growth (biomass) without}

\author{
affecting maturity. (a) Polymorphisms and haplotypes of GmFULa. Bottom inset
} shows the exon-intron structure of GmFULa and the location of polymorphisms, where red, green and blue bars indicate $5^{\prime}$ UTR, CDS and 3' UTR, respectively, and blue and black lines respectively indicate missense and synonymous variants. The upper panel shows the haplotypes of GmFULa. Reference alleles are in green; alternative alleles are in purple. (b) Distribution of GmFULa haplotypes. The upper panel shows the general distribution of GmFULa haplotypes detected in all soybeans.

The bottom panel shows the distribution of $\mathrm{HO}$ and $\mathrm{HO} 2$ haplotypes in cultivars from Northeast China (NE), cultivars from the Huang-Huai-Hai valley region and South China (HS), and wild soybeans (Wild). (c) Verification of GmFULa overexpression in transgenic lines by real-time quantitative PCR analysis. GmActin was used as an internal control. Values are given as mean $\pm \mathrm{SE}$ of three biological replicates with letters showing if there is a significant difference between groups (One-Way ANOVA; Tukey HSD test at, <0.05). (d) The growth stages of transgenic soybean lines and control soybean ZGDD. V1, V2, R1 and R8 are soybean growth stages of one unrolled trifoliolate leaf, two unrolled trifoliolate leaves, beginning flowering and full maturity, respectively. ZGDD: control soybean Zigongdongdou (transgenic receptor). FU64, FU123 and FU160 are independent transgenic lines overexpressing GmFULa. The 
data represents the mean $\pm S E$ of $\geq 20$ biological replicates with letters showing if

546 there is a significant difference between groups (One-Way ANOVA; Tukey HSD test at,

$547<0.05)$

Figure 2 Overexpression of GmFULa enhances biomass accumulation. (a) Transgenic

549 plants overexpressing GmFULa reach the same growth stage as control soybean

550 ZGDD at 3 days after germination (DAG). (b) Transgenic plants overexpressing

551 GmFULa have bigger unifoliolate leaves than control soybean ZGDD at 7 DAG. (c)

552 Transgenic plants overexpressing GmFULa have one more trifoliolate leaf than

553 control soybean ZGDD at 15 DAG. (d-f) Cotyledons and leaves of transgenic plants

554 overexpressing GmFULa and control soybean ZGDD at 3 (d), 7 (e), and 15 (f) DAG. (g)

555 Overexpression of GmFULa promotes the accumulation of dry biomass at 3, 7 and 15

556 DAG ( $n=5)$. ZGDD: control soybean Zigongdongdou (transgenic receptor). FU64,

557 FU123 and FU160 are independent transgenic lines overexpressing GmFULa. Bar

558 indicates $5 \mathrm{~cm}$. The data represents the mean \pm SE of five biological replicates with

559 letters showing if there is a significant difference between groups (One-Way ANOVA;

560 Tukey HSD test at, <0.05).

561 Figure 3. Overexpression of GmFULa promotes soybean yield. (a) Representative

562 seed sizes of transgenic lines and control. (b-g) Yield-related traits branch number

563 (b), plant height (c), node number (d), pod number (e), and seed number (f) and 564 overall yield $(\mathrm{g})$ of transgenic lines and control grown under natural field conditions.

565 The plants were grown in a field in Sanya, Hainan province, China. The data 566 represents the mean \pm SE from three replicates (ten plants per replicate) with letters 

$587<0.05)$.

showing if there is a significant difference between groups (One-Way ANOVA; Tukey HSD test at, <0.05). ZGDD: control soybean Zigongdongdou (transgenic receptor). FU64, FU123 and FU160 are independent transgenic lines overexpressing GmFULa.

Figure 4. Overexpression of GmFULa increases the number and size of leaves cell. (a) and (b) Leaf sampling method for cell morphology analysis. (c) Cell morphology of mesophyll of cross section in wildtype and transgenic plants. (d) Cell morphology of leaf vein cross section in wildtype and transgenic plants. (e-k) Comparison of cell morphology in different tissues.ZGDD: control soybean Zigongdongdou (transgenic receptor). FU160 is the typical transgenic line overexpressing GmFULa. The data represent mean $\pm \mathrm{SE}$ from three replicates. ${ }^{*}, \mathrm{p}<0.05 ;{ }^{* *}, \mathrm{p}<0.01$ (Student's $t$-test). Scale bars are $250 \mu \mathrm{m}$.

Figure 5. Overexpression of GmFULa increases the content of chlorophyll and promotes the rate of photosynthesis. (a) Chlorophyll content in control and transgenic plant leaves. The data represent mean \pm SE from three biological replicates with letters showing if there is a significant difference between groups (One-Way ANOVA; Tukey HSD test at, <0.05). (b) Photosynthesis in control and transgenic plants. ZGDD: control soybean Zigongdongdou transgenic receptor. FU64, FU123 and FU160 are the independent transgenic lines overexpressing GmFULa. The data represent mean \pm SE from fifteen biological replicates with letters showing if there is a significant difference between groups (One-Way ANOVA; Tukey HSD test at,

Figure 6. GmFULa regulates sucrose synthases and transporters to increase soluble 
sugar content of soybean leaves and seeds. (a) Relative content of soluble

590 carbohydrate in leaves of transgenic lines and control plant. (b) Relative content of 591 soluble carbohydrate in seeds of transgenic lines and control plant. (c) The activity of 592 sucrose synthase in leaves of transgenic lines and control plant. (d) Subcellular 593 localization of GmFULa in soybean protoplasts. GFP and GmFULa-GFP fusions under 594 the control of the CaMV35S promoter were transiently expressed in soybean 595 protoplasts. Bar=10 $\mu \mathrm{m}$. (e) Relative expression levels of GmSUSs and GmSUTs in 596 transgenic lines and the control. Soybean GmActin was used as an internal control. (a)-(c) and (e) The data represent mean \pm SE from three biological replicates with 598 letters showing if there is a significant difference between groups (One-Way ANOVA; 599 Tukey HSD test at, <0.05). (f) GmFULa binds to the GmSUS12 and GmSUT5 600 promoters. Schematic diagram of the 2,000 bp GmSUS12 and GmSUT5 promoter 601 regions showed three and seven CArG boxes, respectively. EMSA assay testing the 602 binding of GmFULa to the GmSUS12 and GmSUT5 promoter fragments. Two 40 bp 603 single strand oligonucleotide probes containing CArG box motif with 16 bp flanking 604 sequences were synthesized and labeled with biotin. + and - indicate the presence 605 and absence of the corresponding probe or protein. ZGDD: control soybean 606 Zigongdongdou (transgenic receptor). FU64, FU123 and FU160 are the independent 607 transgenic lines overexpressing GmFULa.

Figure 7. Working model for regulation of soybean vegetative growth, cell 609 development, and yield by GmFULa. GmFULa regulates both the sucrose synthases 610 (SUS) and the sucrose transporters to synchronize the sucrose biosynthesis (energy 
611 generation and assimilation) in the source organ (leaf) and the sucrose 612 transportation (energy transportation) to the sink organ (pod) to finally promote 613 yield.

614 


\section{References}

Ainsworth EA, Rogers A, Nelson R, Long SP. (2004). Testing the "source-sink" hypothesis of down-regulation of photosynthesis in elevated $\left[\mathrm{CO}_{2}\right]$ in the field with single gene substitutions in Glycine max. Agric For Meteorol 122:85-94.

Alvarez-Buylla ER, García-Ponce B, Sánchez MdIP, Espinosa-Soto C, García-Gómez ML, Piñeyro-Nelson A, Garay-Arroyo A. (2019). MADS-box genes underground becoming mainstream: plant root developmental mechanisms. New Phytol 223:1143-1158.

Arrese-Igor C, Gonzalez E, Gordon A, Minchin F, Galvez L, Royuela M, Cabrerizo P, Aparicio-Tejo P. (1999). Sucrose synthase and nodule nitrogen fixation under drought and environmental stresses. Symbiosis 27:189-212.

Balanza V, Martinez-Fernandez I, Sato S, Yanofsky MF, Ferrandiz C. (2019). Inflorescence meristem fate is dependent on seed development and FRUITFULL in Arabidopsis thaliana. Front Plant Sci 10:1622.

Barker L, Kühn C, Weise A, Schulz A, Gebhardt C, Hirner B, Hellmann H, Schulze W, Ward JM, Frommer WB. (2000). SUT2, a putative sucrose sensor in sieve elements. Plant Cell 12:1153-64.

Bemer M, Karlova R, Ballester AR, Tikunov YM, Bovy AG, Wolters-Arts M, Rossetto PdB, Angenent GC, de Maagd RA. (2012). The tomato FRUITFULL homologs TDR4/FUL1 and MBP7/FUL2 regulate ethylene-independent aspects of fruit ripening. Plant Cell 24:4437-51.

Bemer M, van Mourik H, Muino JM, Ferrandiz C, Kaufmann K, Angenent GC. (2017). FRUITFULL controls SAUR10 expression and regulates Arabidopsis growth and architecture. J Exp Bot 68:3391-3403.

Berbel A, Ferrándiz C, Hecht V, Dalmais M, Lund OS, Sussmilch FC, Taylor SA, Bendahmane A, Ellis THN, Beltrán JP, Weller JL, Madueño F. (2012). VEGETATIVE1 is essential for development of the compound inflorescence in pea. Nat Commun $3: 797$.

Bianchi JS, Quijano A, Gosparini CO, Morandi EN. (2020). Changes in leaflet shape and seeds per pod modify crop growth parameters, canopy light environment, and yield components in soybean. Crop J 8:351-364.

Breia R, Conde A, Conde C, Fortes AM, Granell A, Gerós H. (2020). VvERD6l13 is a grapevine sucrose transporter highly up-regulated in response to infection by Botrytis cinerea and Erysiphe necator. Plant Physiol Bioch 154:508-516.

Busch, F.A., (2018). Photosynthetic gas exchange in land plants at the leaf level. In: Covshoff, S. eds. Photosynthesis: Methods and Protocols. New York, NY: Springer New York; 2018:25-44.

BUTTERY BR, BUZZELL RI. (1977). The relationship between chlorophyll content and rate of photosynthesis in soybeans. Can J Plant Sci 57:1-5.

Chen L, Nan H, Kong L, Yue L, Yang H, Zhao Q, Fang C, Li H, Cheng Q, Lu S, Kong F, Liu B, Dong L. (2020). Soybean AP1 homologs control flowering time and plant 
height. J Integr Plant Biol 62:1868-1879.

Di Marzo M, Herrera-Ubaldo H, Caporali E, Novak O, Strnad M, Balanza V, Ezquer I, Mendes MA, de Folter S, Colombo L. (2020). SEEDSTICK controls Arabidopsis fruit size by regulating cytokinin levels and FRUITFULL. Cell Rep 30:2846-2857.e3.

Ferrandiz C, Liljegren SJ, Yanofsky MF. (2000). Negative regulation of the SHATTERPROOF genes by FRUITFULL during Arabidopsis fruit development. Science 289:436-8.

Frearson EM, Power JB, Cocking EC. (1973). The isolation, culture and regeneration of Petunia leaf protoplasts. Dev Biol 33:130-7.

Gessler A. (2021). Sucrose synthase - an enzyme with a central role in the source-sink coordination and carbon flow in trees. New Phytol 229:8-10.

Gu Q, Ferrandiz C, Yanofsky MF, Martienssen R. (1998). The FRUITFULL MADS-box gene mediates cell differentiation during Arabidopsis fruit development. Development 125:1509-17.

Hu D, Zhang H, Du Q, Hu Z, Yang Z, Li X, Wang J, Huang F, Yu D, Wang H, Kan G. (2020). Genetic dissection of yield-related traits via genome-wide association analysis across multiple environments in wild soybean (Glycine soja Sieb. and Zucc.). Planta 251:39.

Huang JW, Chen JT, Yu WP, Shyur LF, Wang AY, Sung HY, Lee PD, Su JC. (1996). Complete structures of three rice sucrose synthase isogenes and differential regulation of their expressions. Biosci Biotechnol Biochem 60:233-9.

Huang Y, Wang L, Hu S, Luo X, Cao Y. (2020). Overexpression of the bamboo sucrose synthase gene (BeSUS5) improves cellulose production, cell wall thickness and fiber quality in transgenic poplar. Tree Genetics and Genomes 16:75.

Jaakola $L$, Poole $M$, Jones $M O$, Kämäräinen-Karppinen T, Koskimäki JJ, Hohtola $A$, Häggman H, Fraser PD, Manning K, King GJ, Thomson H, Seymour GB. (2010). A SQUAMOSA MADS box gene involved in the regulation of anthocyanin accumulation in bilberry fruits. Plant Physiol 153:1619-29.

Jaudal M, Zhang L, Che C, Putterill J. (2015). Three Medicago MtFUL genes have distinct and overlapping expression patterns during vegetative and reproductive development and 35S:MtFULb accelerates flowering and causes a terminal flower phenotype in Arabidopsis. Front Genet 6:50.

Jeong N, Suh SJ, Kim M-H, Lee S, Moon J-K, Kim HS, Jeong S-C. (2012). Ln is a key regulator of leaflet shape and number of seeds per pod in soybean. Plant Cell 24:4807-18.

Jia Z, Jiang B, Gao X, Yue Y, Fei Z, Sun H, Wu C, Sun S, Hou W, Han T. (2015). GmFULa, a FRUITFULL homolog, functions in the flowering and maturation of soybean. Plant Cell Rep 34:121-32.

Jiang B, Zhang S, Song W, Khan MAA, Sun S, Zhang C, Wu T, Wu C, Han T. (2019). Natural variations of $F T$ family genes in soybean varieties covering a wide range of maturity groups. Bmc Genomics 20:230.

Koester RP, Nohl BM, Diers BW, Ainsworth EA. (2016). Has photosynthetic capacity increased with 80 years of soybean breeding? An examination of historical soybean cultivars. Plant Cell Environ 39:1058-67. 
Lam H-M, Xu X, Liu X, Chen W, Yang G, Wong F-L, Li M-W, He W, Qin N, Wang B, Li J, Jian M, Wang J, Shao G, Wang J, Sun SS-M, Zhang G. (2010). Resequencing of 31 wild and cultivated soybean genomes identifies patterns of genetic diversity and selection. Nat Genet 42:1053-9.

Langdale JA, Lane B, Freeling M, Nelson T. (1989). Cell lineage analysis of maize bundle sheath and mesophyll cells. Dev Biol 133:128-39.

Li S, Chen K, Grierson D. (2019). A critical evaluation of the role of ethylene and MADS transcription factors in the network controlling fleshy fruit ripening. New Phytol 221:1724-1741.

Lin K, Liu X, Sun S, Chen L, Han T, Hou W. (2015). Salt tolerance analysis of TaNHX2 over-expression transgenic soybean. Scientia Agricultura Sinica 48:3998-4007.

Liu Y, Zhang D, Ping J, Li S, Chen Z, Ma J. (2016). Innovation of a regulatory mechanism modulating semi-determinate stem growth through artificial selection in soybean. Plos Genet 12:e1005818.

Ma G, Zou Q, Shi X, Tian D, Sheng Q. (2019). Ectopic expression of the AaFUL1 gene identified in Anthurium andraeanum affected floral organ development and seed fertility in tobacco. Gene 696:197-205.

Maheepala DC, Emerling CA, Rajewski A, Macon J, Strahl M, Pabon-Mora N, Litt A. (2019). Evolution and diversification of FRUITFULL genes in Solanaceae. Front Plant Sci 10:43.

Mandel MA, Yanofsky MF. (1995). The Arabidopsis AGL8 MADS box gene is expressed in inflorescence meristems and is negatively regulated by APETALA1. Plant Cell 7:1763-71.

Müller BM, Saedler H, Zachgo S. (2001). The MADS-box gene DEFH28 from Antirrhinum is involved in the regulation of floral meristem identity and fruit development. Plant J 28:169-79.

Nguyen CX, Paddock KJ, Zhang Z, Stacey MG. (2021). GmKIX8-1 regulates organ size in soybean and is the causative gene for the major seed weight QTL qSw17-1. New Phytol 229:920-934.

Paz MM, Shou H, Guo Z, Zhang Z, Banerjee AK, Wang K. (2004). Assessment of conditions affecting Agrobacterium-mediated soybean transformation using the cotyledonary node explant. Euphytica 136:167-179.

Van Roekel RJ, Purcell LC, Salmerón M. (2015). Physiological and management factors contributing to soybean potential yield. Field Crop Res 182:86-97.

Sayama T, Tanabata T, Saruta M, Yamada T, Anai T, Kaga A, Ishimoto M. (2017). Confirmation of the pleiotropic control of leaflet shape and number of seeds per pod by the Ln gene in induced soybean mutants. Breeding science 67:363-369.

Sehra B, Franks RG. (2017). Redundant CArG box cis-motif activity mediates SHATTERPROOF2 transcriptional regulation during Arabidopsis thaliana gynoecium development. Front Plant Sci 8:1712.

Singsaas EL, Ort DR, Delucia EH. (2004). Elevated $\mathrm{CO}_{2}$ effects on mesophyll conductance and its consequences for interpreting photosynthetic physiology. Plant Cell Environ 27:41-50.

Slattery RA, Grennan AK, Sivaguru M, Sozzani R, Ort DR. (2016). Light sheet 
microscopy reveals more gradual light attenuation in light-green versus darkgreen soybean leaves. J Exp Bot 67:4697-709.

Slattery RA, VanLoocke A, Bernacchi CJ, Zhu X-G, Ort DR. (2017). Photosynthesis, light use efficiency, and yield of reduced-chlorophyll soybean mutants in field conditions. Front Plant Sci 8:549.

Taylor SC, Nadeau K, Abbasi M, Lachance C, Nguyen M, Fenrich J. (2019). The ultimate qPCR experiment: producing publication quality, reproducible data the first time. Trends Biotechnol 37:761-774.

Wang $D$, Liu $H$, Wang $H$, Zhang $P$, Shi C. (2020). A novel sucrose transporter gene IbSUT4 involves in plant growth and response to abiotic stress through the ABFdependent ABA signaling pathway in Sweetpotato. Bmc Plant Biol 20:157.

Wang S, Lu G, Hou Z, Luo Z, Wang T, Li H, Zhang J, Ye Z. (2014). Members of the tomato FRUITFULL MADS-box family regulate style abscission and fruit ripening. J Exp Bot 65:3005-14.

Wang X, Li Y, Zhang H, Sun G, Zhang W, Qiu L. (2015). Evolution and association analysis of GmCYP78A10 gene with seed size/weight and pod number in soybean. Mol Biol Rep 42:489-96.

Williams LE, Lemoine R, Sauer N. (2000). Sugar transporters in higher plants--a diversity of roles and complex regulation. Trends Plant Sci 5:283-90.

Xiao X, Tang C, Fang Y, Yang M, Zhou B, Qi J, Zhang Y. (2014). Structure and expression profile of the sucrose synthase gene family in the rubber tree: indicative of roles in stress response and sucrose utilization in the laticifers. FEBS J 281:291-305.

Xu C, Yin Y, Cai R, Wang P, Ni Y, Guo J, Chen E, Cai T, Cui Z, Liu T, Yang D, Wang Z. (2013). Responses of photosynthetic characteristics and antioxidative metabolism in winter wheat to post-anthesis shading. Photosynthetica 51:139150.

Xu S-M, Brill E, Llewellyn DJ, Furbank RT, Ruan Y-L. (2012). Overexpression of a potato sucrose synthase gene in cotton accelerates leaf expansion, reduces seed abortion, and enhances fiber production. Mol Plant 5:430-41.

Yan L, Hofmann N, Li S, Ferreira ME, Song B, Jiang G, Ren S, Quigley C, Fickus E, Cregan P, Song Q. (2017). Identification of QTL with large effect on seed weight in a selective population of soybean with genome-wide association and fixation index analyses. Bmc Genomics 18:529.

Yao T, Park BS, Mao H-Z, Seo JS, Ohama N, Li Y, Yu N, Mustafa NFB, Huang C-H, Chua $\mathrm{N}-\mathrm{H}$. (2019). Regulation of flowering time by SPL10/MED25 module in Arabidopsis. New Phytol 224:493-504.

Yoo S-D, Cho Y-H, Sheen J. (2007). Arabidopsis mesophyll protoplasts: a versatile cell system for transient gene expression analysis. Nat Protoc 2:1565-72.

Yue, Y., Liu, N., Jiang, B., Li, M., Wang, H., Jiang, Z., Pan, H., Xia, Q., Ma, Q., Han, T., Nian, H., 2017. A single nucleotide deletion in $J$ encoding GmELF3 confers long juvenility and is associated with adaption of tropic soybean. Mol Plant 10, 656658.

Zhang S, Lu S, Yi S, Han H, Zhou Q, Cai F, Bao M, Liu G. (2019a). Identification and characterization of FRUITFULL-like genes from Platanus acerifolia, a basal 
eudicot tree. Plant Sci 280:206-218.

Zhang T, Wu T, Wang L, Jiang B, Zhen C, Yuan S, Hou W, Wu C, Han T, Sun S. (2019b). A combined linkage and GWAS analysis identifies QTLs linked to soybean seed $790 \quad$ protein and oil content. Int J Mol Sci 20:5915.

792

Zhang W, Fan S, Pang C, Wei H, Ma J, Song M, Yu S. (2013). Molecular cloning and function analysis of two SQUAMOSA-like MADS-box genes from Gossypium hirsutum L. J Integr Plant Biol 55:597-607.

Zhao J, Jiang L, Che G, Pan Y, Li Y, Hou Y, Zhao W, Zhong Y, Ding L, Yan S, Sun C, Liu R, Yan L, Wu T, Li X, Weng Y, Zhang X. (2019). A functional allele of CsFUL1 regulates fruit length through repressing CSSUP and inhibiting auxin transport in Cucumber. Plant Cell 31:1289-1307.

Zhou Z, Jiang Y, Wang Z, Gou Z, Lyu J, Li W, Yu Y, Shu L, Zhao Y, Ma Y, Fang C, Shen Y, Liu T, Li C, Li Q, Wu M, Wang M, Wu Y, Dong Y, Wan W, Wang X, Ding Z, Gao Y, Xiang H, Zhu B, Lee S-H, Wang W, Tian Z. (2015). Resequencing 302 wild and cultivated accessions identifies genes related to domestication and improvement in soybean. Nat Biotechnol 33:408-14. 


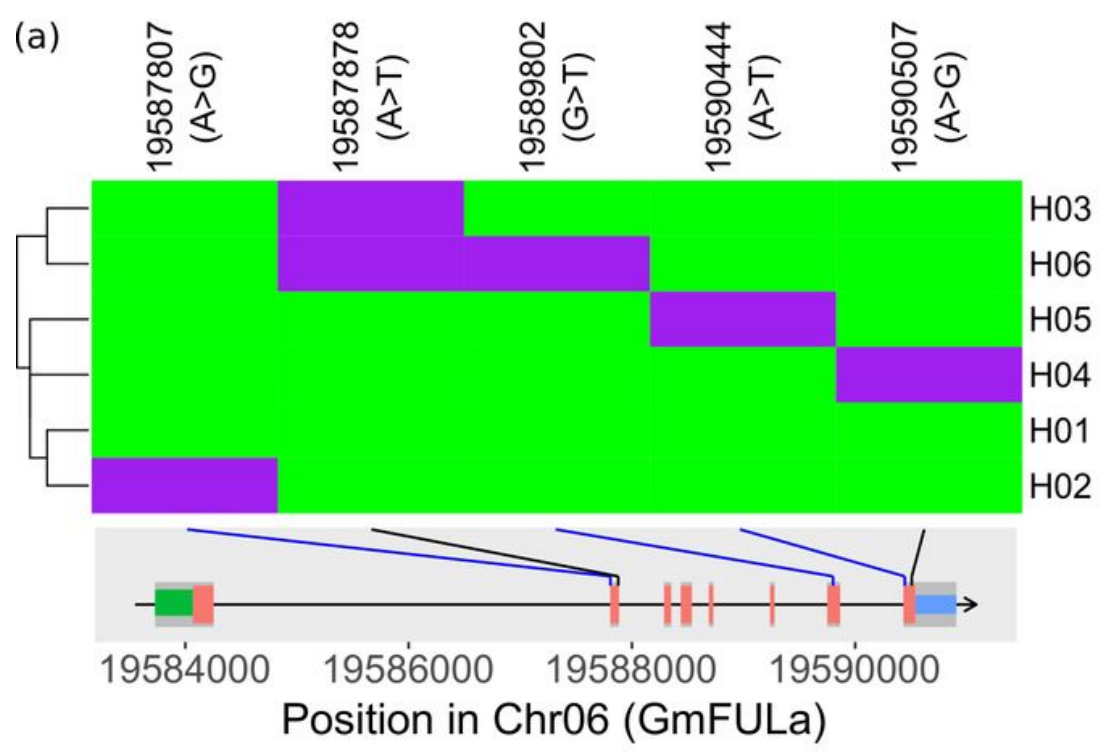

(b)
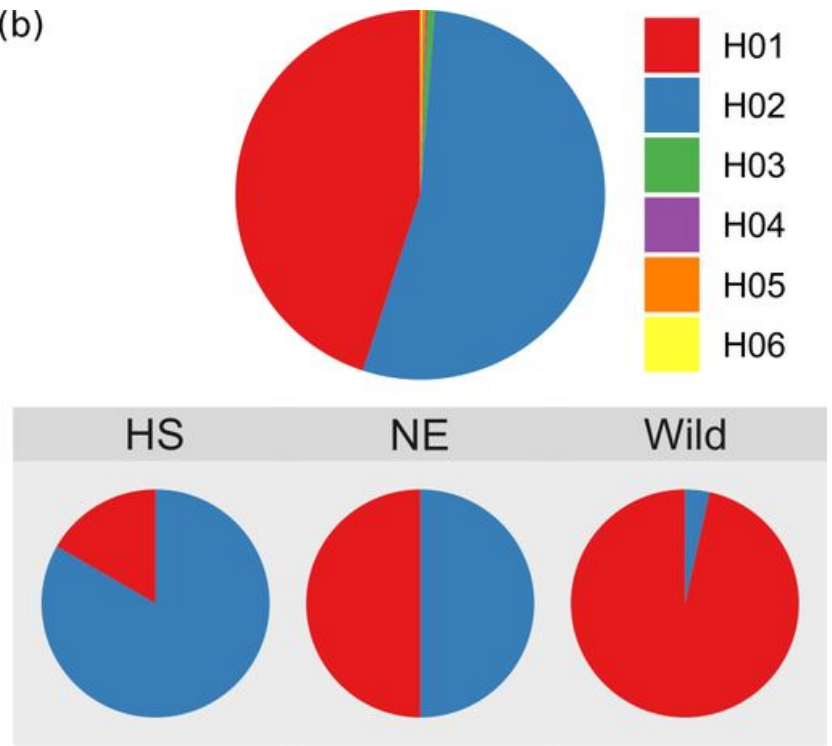

(c)

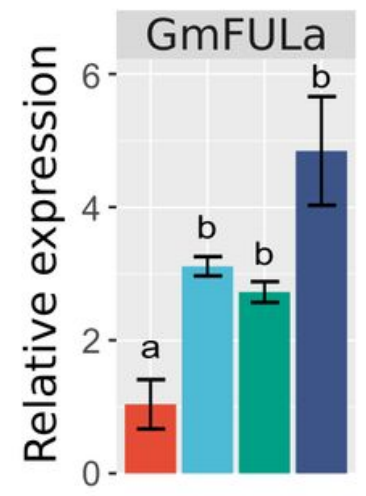

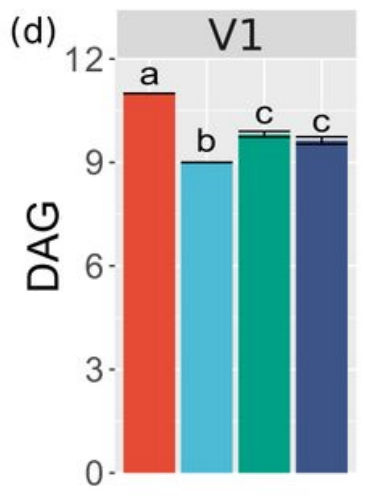

ZGDD

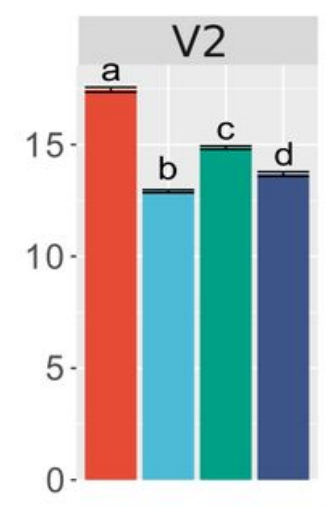

FU123
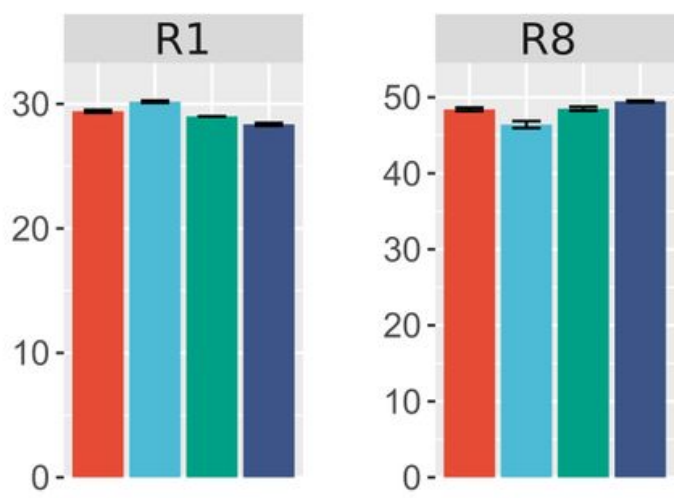

FU64

FU160

\section{Figure 1}

Overexpression of GmFULa promotes vegetative growth (biomass) without affecting maturity. (a) Polymorphisms and haplotypes of GmFULa. Bottom inset shows the exon-intron structure of GmFULa and the location of polymorphisms, where red, green and blue bars indicate 5' UTR, CDS and 3' UTR, respectively, and blue and black lines respectively indicate missense and synonymous variants. The upper panel shows the haplotypes of GmFULa. Reference alleles are in green; alternative alleles are in purple. (b) Distribution of GmFULa haplotypes. The upper panel shows the general distribution of GmFULa haplotypes detected in all soybeans. The bottom panel shows the distribution of $\mathrm{H} 01$ and $\mathrm{H} 02$ haplotypes in cultivars from Northeast China (NE), cultivars from the Huang-Huai-Hai valley region and South China (HS), and wild soybeans (Wild). (c) Verification of GmFULa overexpression in transgenic lines by real-time quantitative PCR analysis. GmActin was used as an internal control. Values are given as mean \pm SE of three biological replicates with letters showing if there is a significant difference between groups (One-Way ANOVA; Tukey HSD test at, <0.05). (d) The growth stages of transgenic soybean lines and control soybean ZGDD. V1, V2, R1 and R8 are soybean growth stages of one unrolled trifoliolate leaf, 
two unrolled trifoliolate leaves, beginning flowering and full maturity, respectively. ZGDD: control soybean Zigongdongdou (transgenic receptor). FU64, FU123 and FU160 are independent transgenic lines overexpressing GmFULa. The data represents the mean \pm SE of $\geq 20$ biological replicates with letters showing if there is a significant difference between groups (One-Way ANOVA; Tukey HSD test at, <0.05).
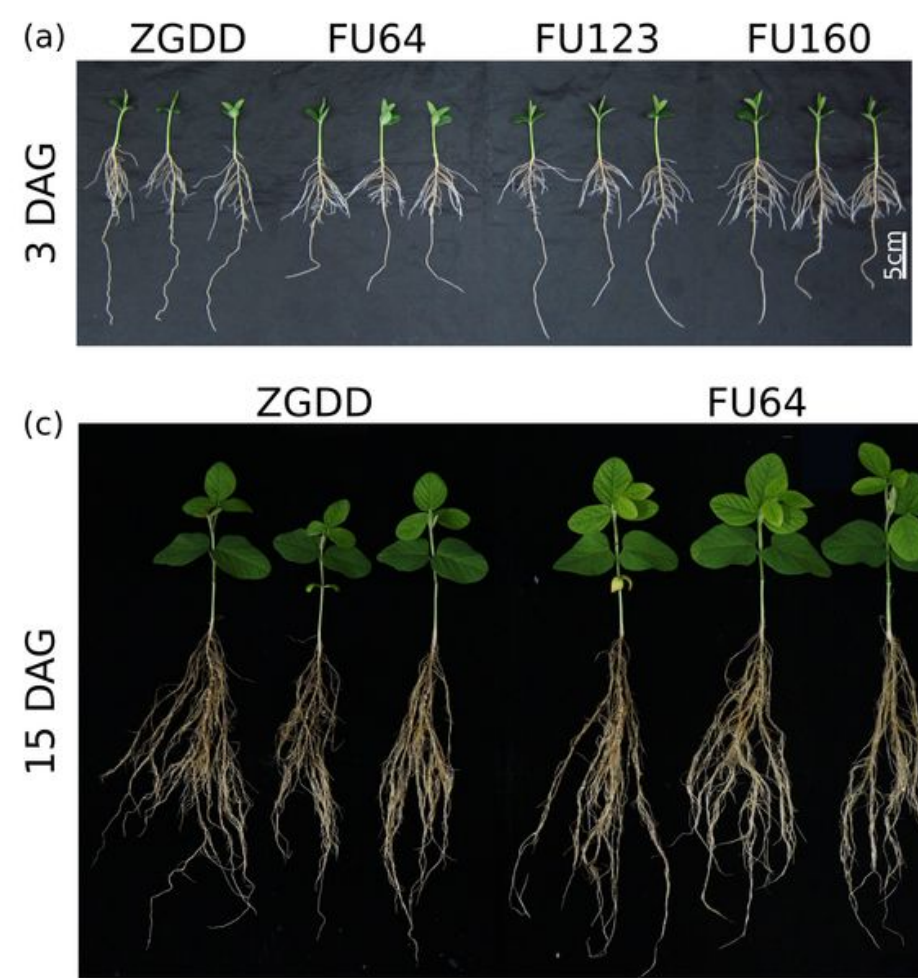

(d) 3 DAG

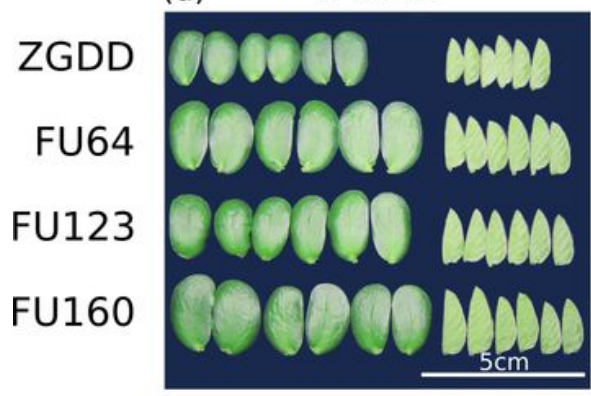

FU64

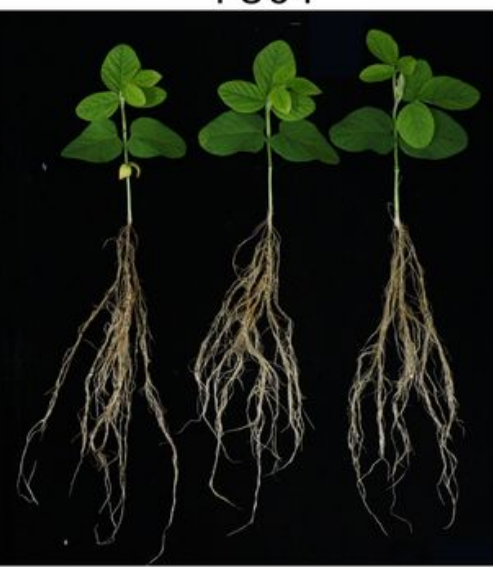

(e)

7 DAG

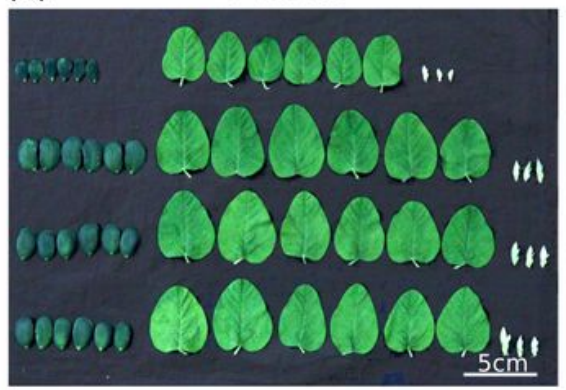

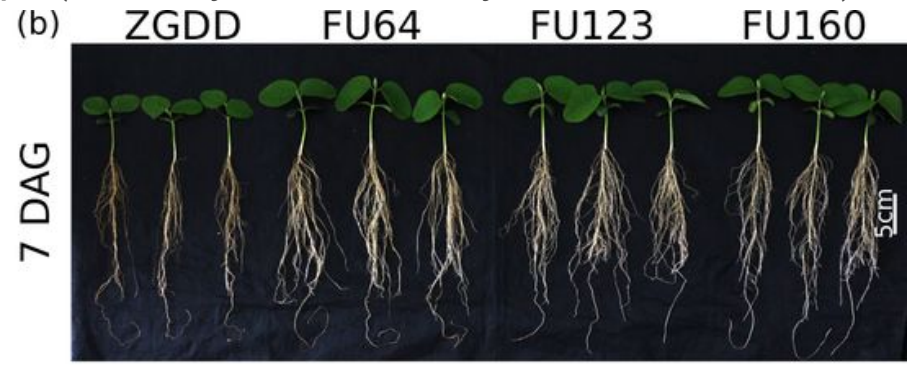

FU123
FU160

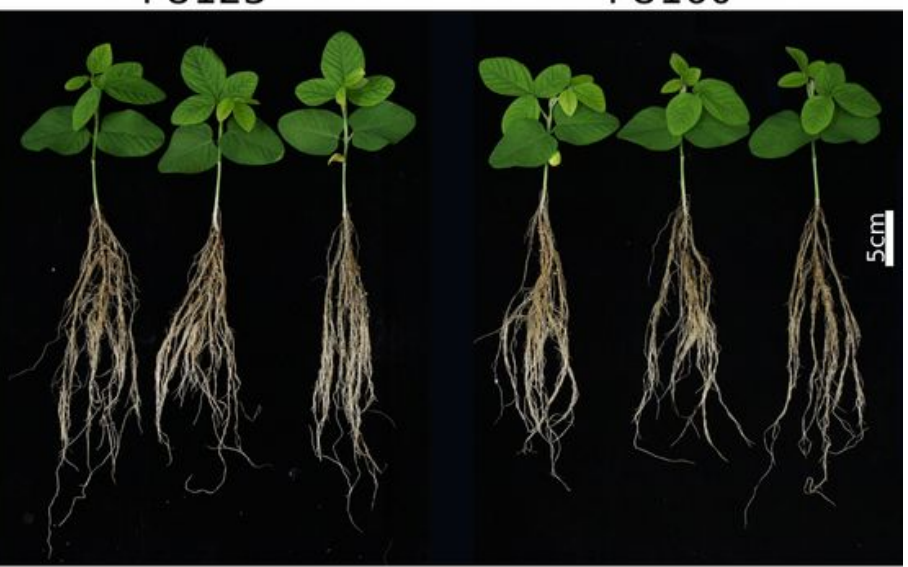

(f)

15 DAG
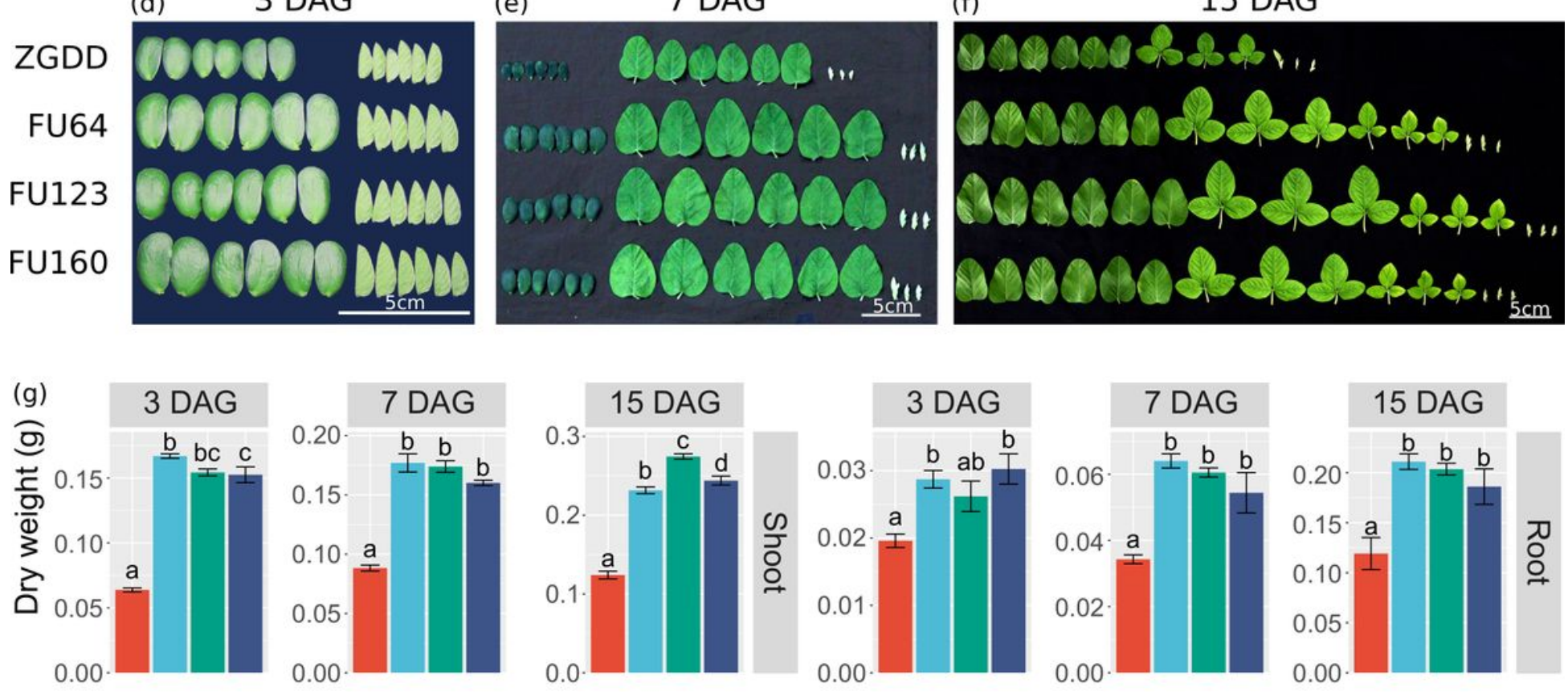

끙

\section{Figure 2}

Overexpression of GmFULa enhances biomass accumulation. (a) Transgenic plants overexpressing GmFULa reach the same growth stage as control soybean ZGDD at 3 days after germination (DAG). (b) 
Transgenic plants overexpressing GmFULa have bigger unifoliolate leaves than control soybean ZGDD at 7 DAG. (c) Transgenic plants overexpressing GmFULa have one more trifoliolate leaf than control soybean ZGDD at 15 DAG. (d-f) Cotyledons and leaves of transgenic plants overexpressing GmFULa and control soybean ZGDD at 3 (d), 7 (e), and 15 (f) DAG. (g) Overexpression of GmFULa promotes the accumulation of dry biomass at 3, 7 and 15 DAG $(n=5)$. ZGDD: control soybean Zigongdongdou (transgenic receptor). FU64, FU123 and FU160 are independent transgenic lines overexpressing GmFULa. Bar indicates $5 \mathrm{~cm}$. The data represents the mean \pm SE of five biological replicates with letters showing if there is a significant difference between groups (One-Way ANOVA; Tukey HSD test at, <0.05). 
(a)
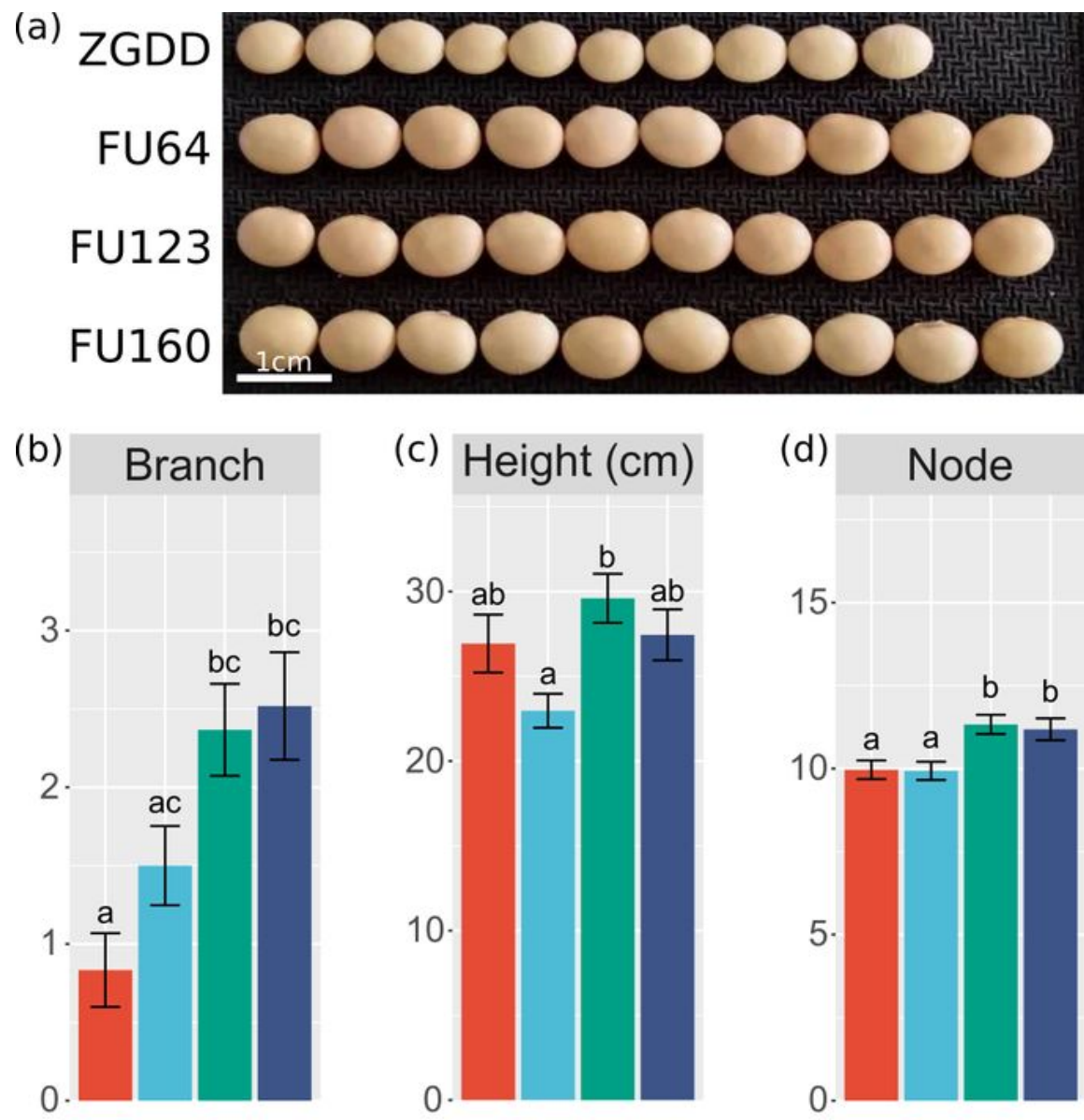

(c) Height $(\mathrm{cm})$

(d) Node

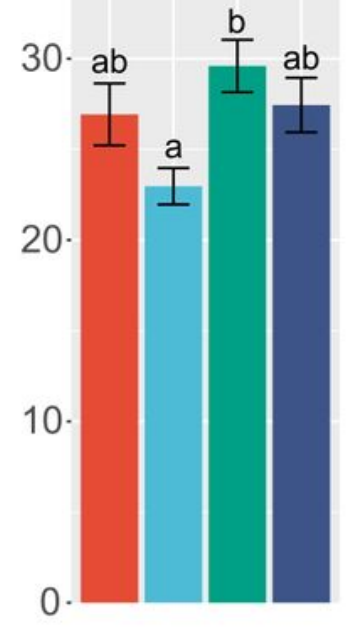

15 .
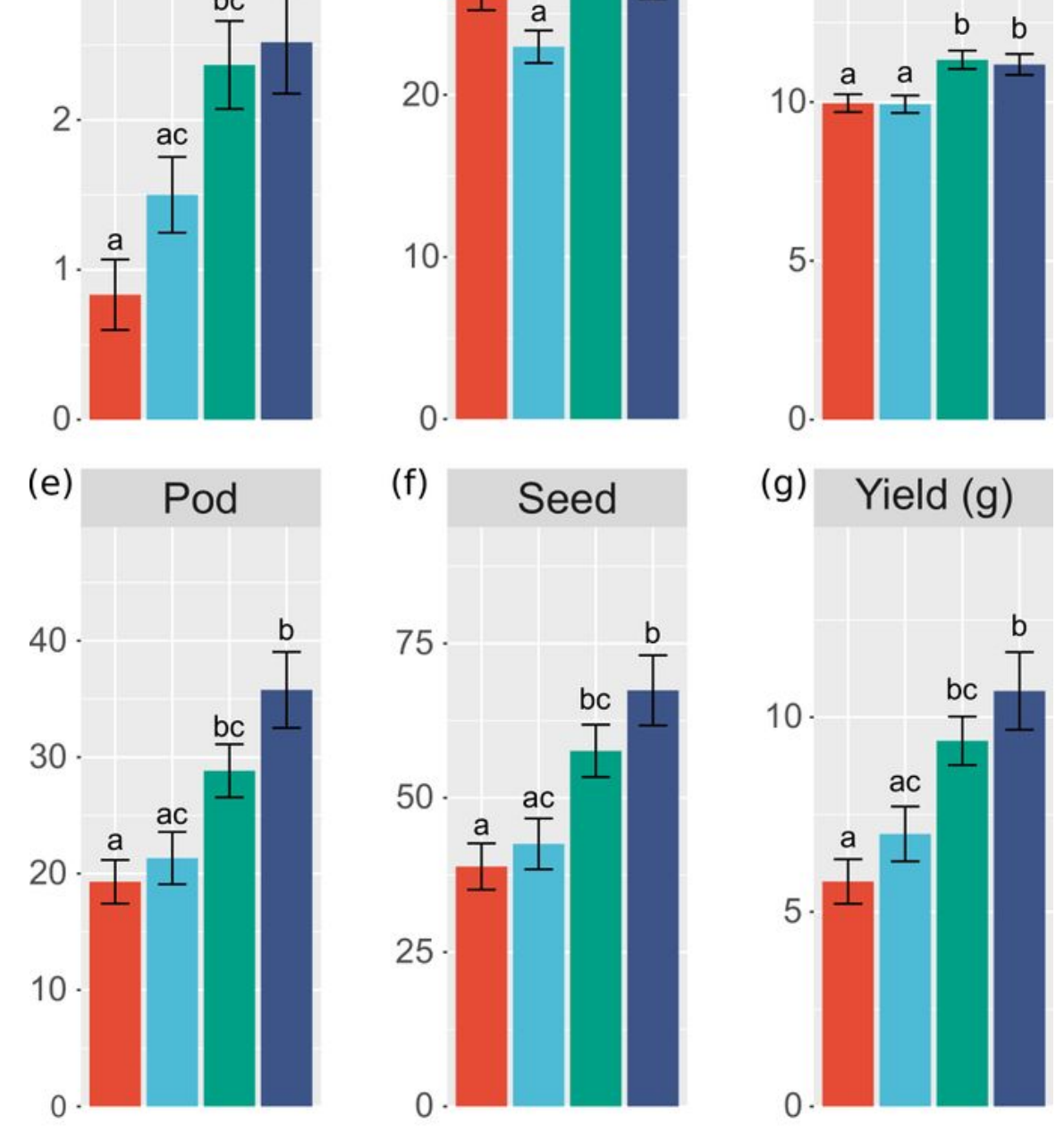

(g) Yield (g)

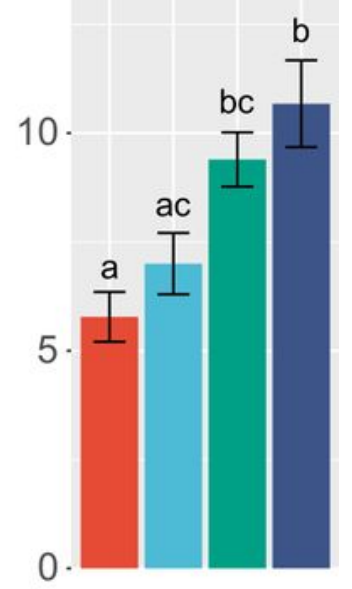

ZGDD

FU64

FU123

FU160

\section{Figure 3}

Overexpression of GmFULa promotes soybean yield. (a) Representative seed sizes of transgenic lines and control. (b-g) Yield-related traits branch number (b), plant height (c), node number (d), pod number (e), and seed number $(\mathrm{f})$ and overall yield $(\mathrm{g})$ of transgenic lines and control grown under natural field conditions. The plants were grown in a field in Sanya, Hainan province, China. The data represents the mean $\pm S E$ from three replicates (ten plants per replicate) with letters showing if there is a significant 
difference between groups (One-Way ANOVA; Tukey HSD test at, <0.05). ZGDD: control soybean Zigongdongdou (transgenic receptor). FU64, FU123 and FU160 are independent transgenic lines overexpressing GmFULa.

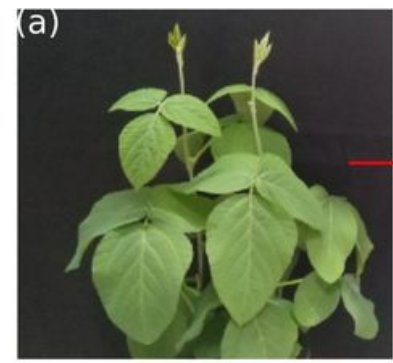

(c)

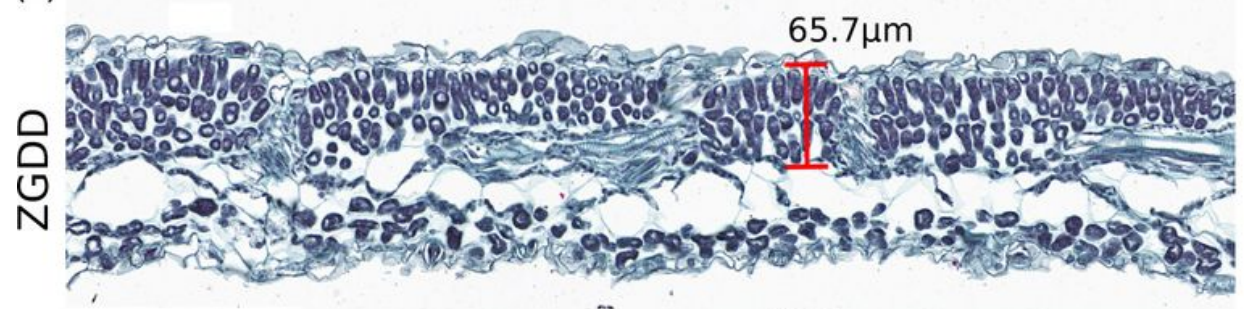

$73.9 \mu \mathrm{m}$
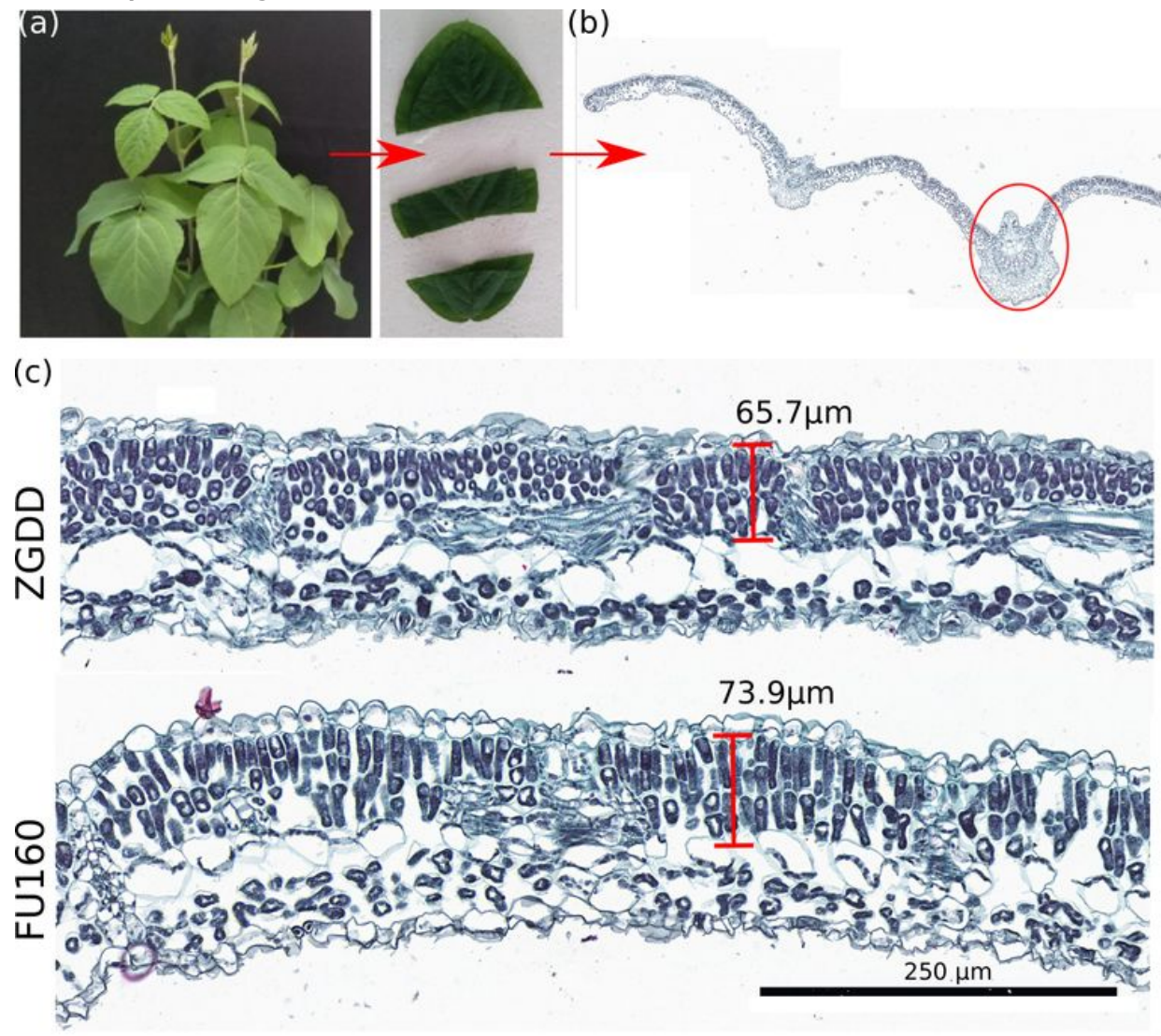

(b)

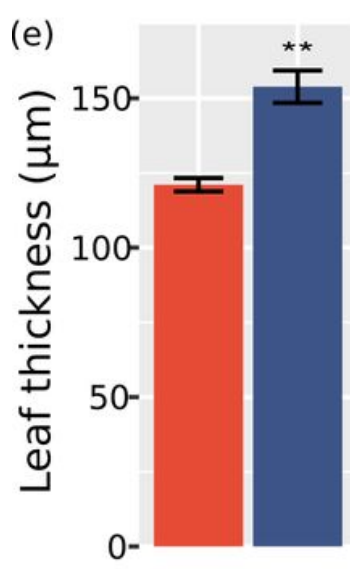

(f)

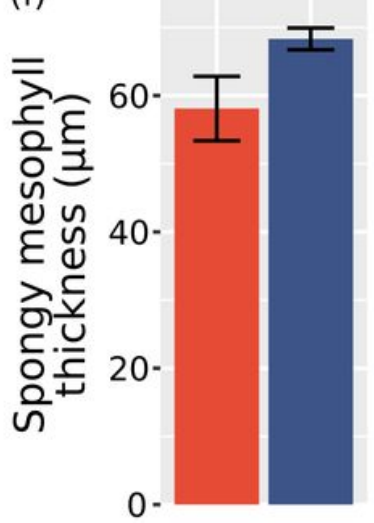

(g) 6-

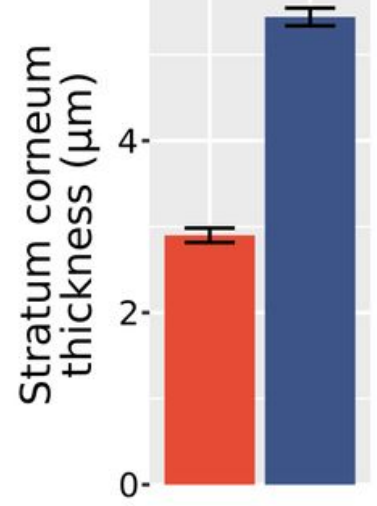

ZGDD

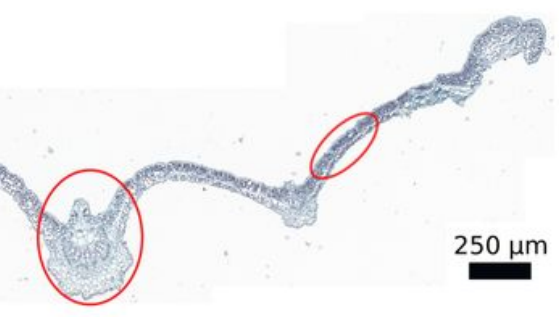

(d)

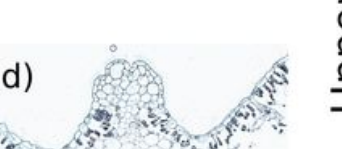

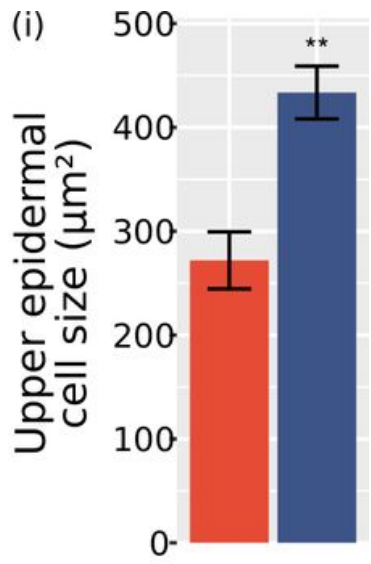

(j)

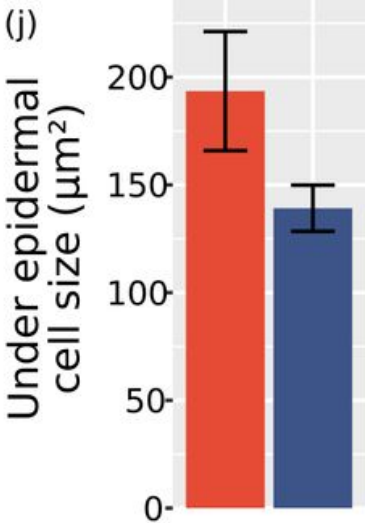

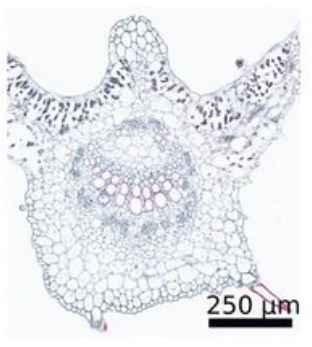

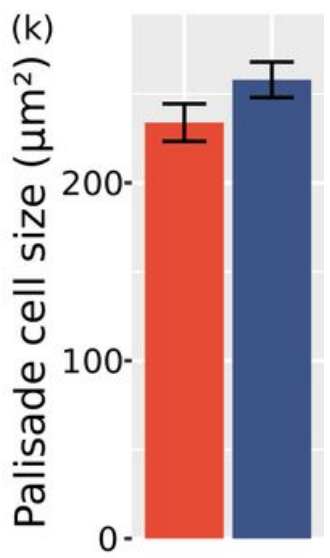

\section{Figure 4}

Overexpression of GmFULa increases the number and size of leaves cell. (a) and (b) Leaf sampling method for cell morphology analysis. (c) Cell morphology of mesophyll of cross section in wildtype and transgenic plants. (d) Cell morphology of leaf vein cross section in wildtype and transgenic plants. (e-k) Comparison of cell morphology in different tissues.ZGDD: control soybean Zigongdongdou (transgenic receptor). FU160 is the typical transgenic line overexpressing GmFULa. The data represent mean \pm SE from three replicates. *, $p<0.05 ; * \star, p<0.01$ (Student's t-test). Scale bars are $250 \mu \mathrm{m}$. 

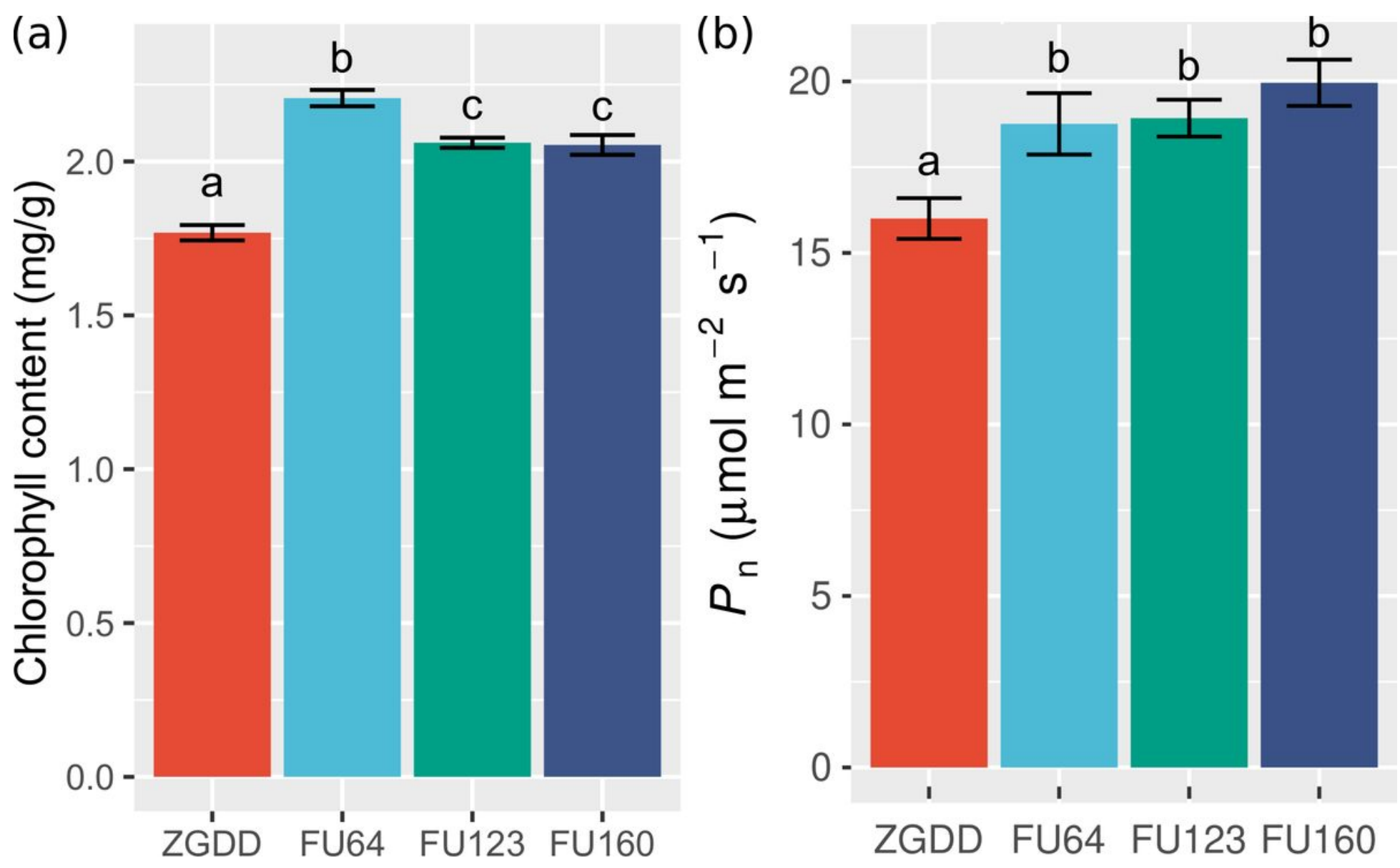

Figure 5

Overexpression of GmFULa increases the content of chlorophyll and promotes the rate of photosynthesis. (a) Chlorophyll content in control and transgenic plant leaves. The data represent mean \pm SE from three biological replicates with letters showing if there is a significant difference between groups (One-Way ANOVA; Tukey HSD test at, <0.05). (b) Photosynthesis in control and transgenic plants. ZGDD: control soybean Zigongdongdou transgenic receptor. FU64, FU123 and FU160 are the independent transgenic lines overexpressing GmFULa. The data represent mean \pm SE from fifteen biological replicates with letters showing if there is a significant difference between groups (One-Way ANOVA; Tukey HSD test at, <0.05). 

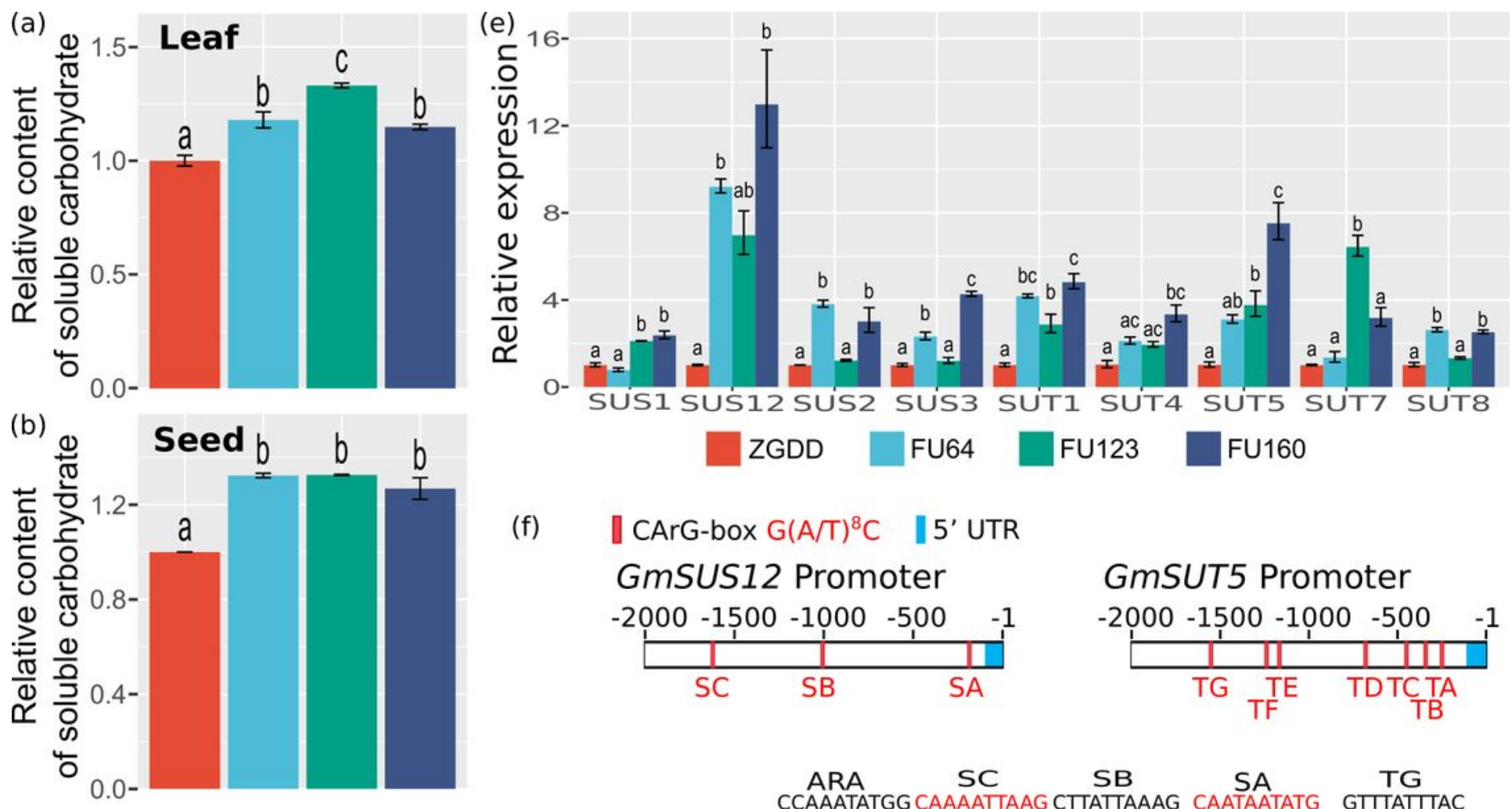

(f) $\|$ CArG-box G(A/T $)^{8} \mathrm{C} \| 5^{\prime}$ UTR
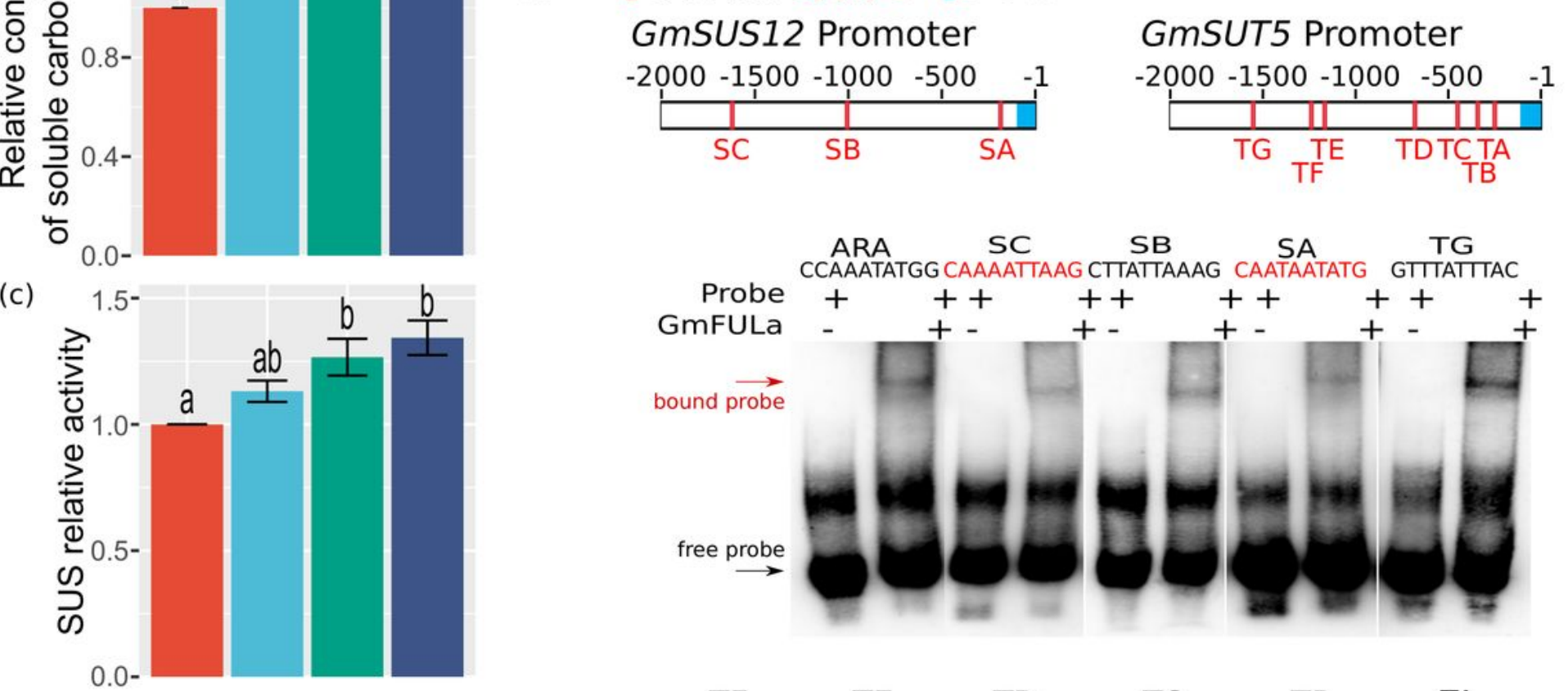

(d)
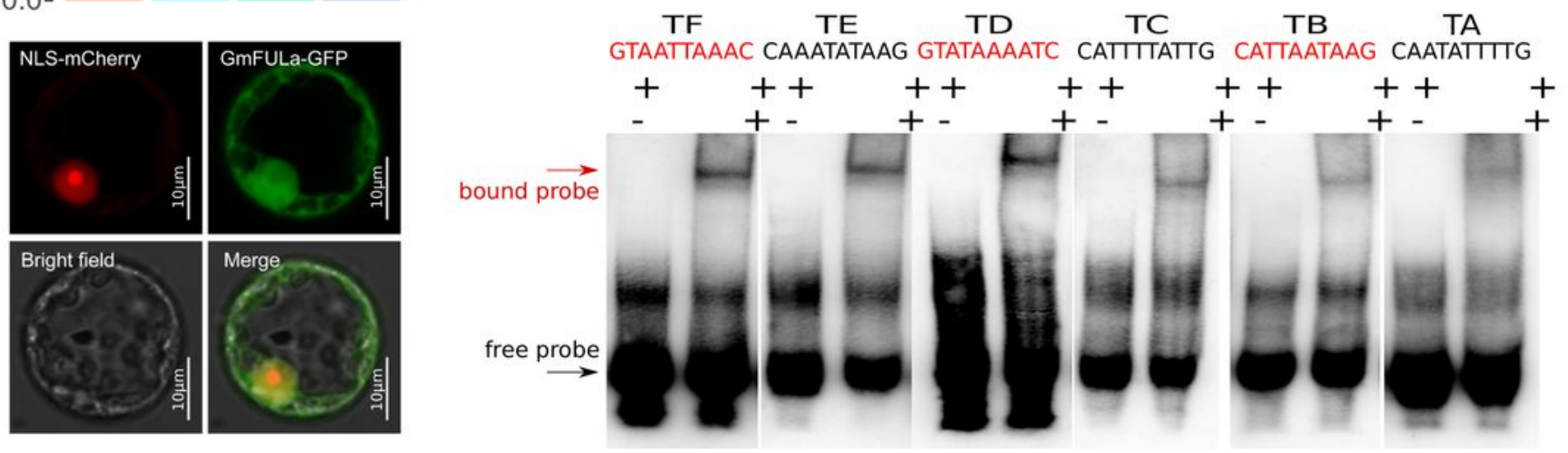

Figure 6

GmFULa regulates sucrose synthases and transporters to increase soluble sugar content of soybean leaves and seeds. (a) Relative content of soluble carbohydrate in leaves of transgenic lines and control plant. (b) Relative content of soluble carbohydrate in seeds of transgenic lines and control plant. (c) The activity of sucrose synthase in leaves of transgenic lines and control plant. (d) Subcellular localization of GmFULa in soybean protoplasts. GFP and GmFULa-GFP fusions under the control of the CaMV35S 
promoter were transiently expressed in soybean protoplasts. Bar=10 $\mu \mathrm{m}$. (e) Relative expression levels of GmSUSs and GmSUTs in transgenic lines and the control. Soybean GmActin was used as an internal control. (a)-(c) and (e) The data represent mean \pm SE from three biological replicates with letters showing if there is a significant difference between groups (One-Way ANOVA; Tukey HSD test at, <0.05). ( $f$ ) GmFULa binds to the GmSUS12 and GmSUT5 promoters. Schematic diagram of the 2,000 bp GmSUS12 and GmSUT5 promoter regions showed three and seven CArG boxes, respectively. EMSA assay testing the binding of GmFULa to the GmSUS12 and GmSUT5 promoter fragments. Two 40 bp single strand oligonucleotide probes containing CArG box motif with 16 bp flanking sequences were synthesized and labeled with biotin. + and - indicate the presence and absence of the corresponding probe or protein. ZGDD: control soybean Zigongdongdou (transgenic receptor). FU64, FU123 and FU160 are the independent transgenic lines overexpressing GmFULa.

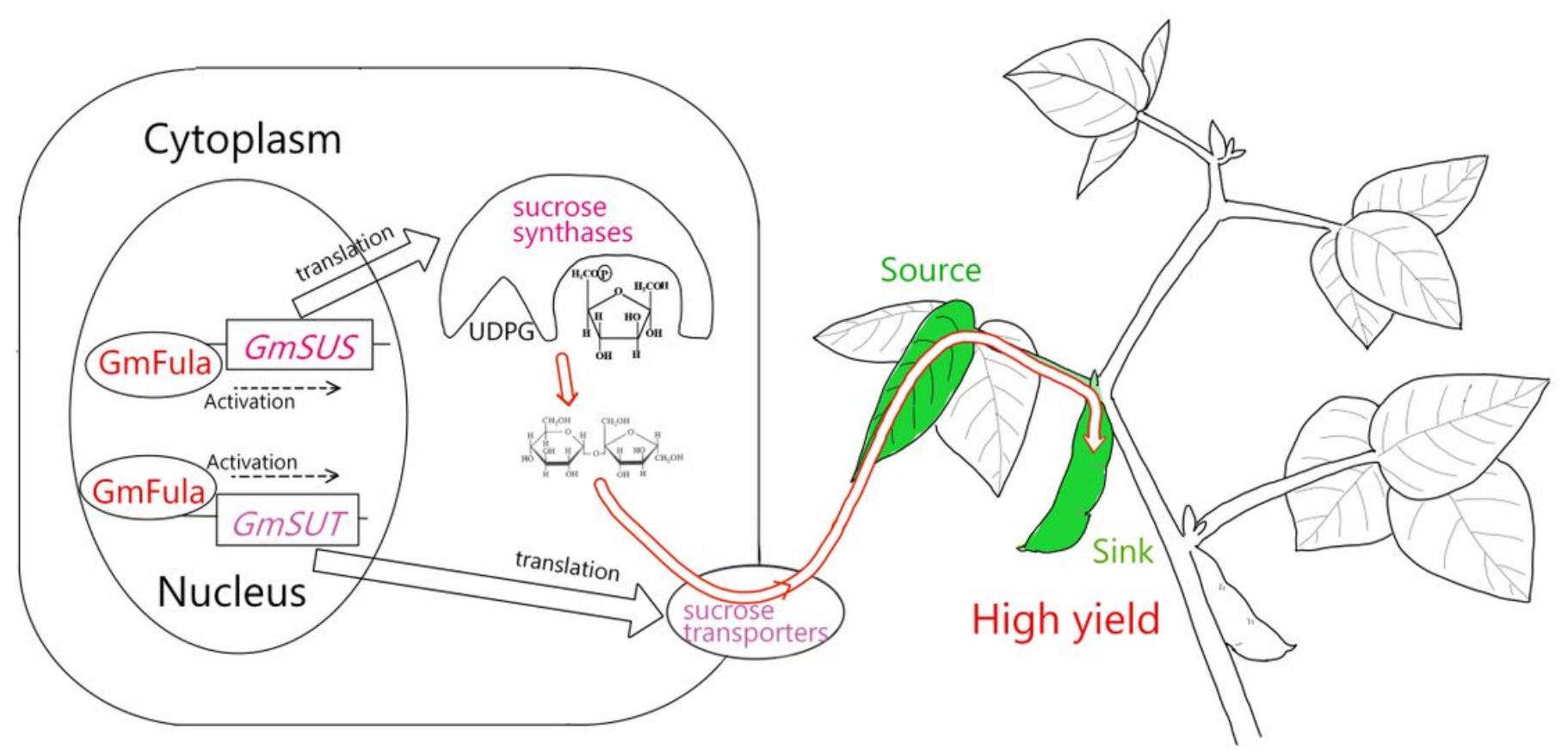

Figure 7

Working model for regulation of soybean vegetative growth, cell development, and yield by GmFULa. GmFULa regulates both the sucrose synthases (SUS) and the sucrose transporters to synchronize the sucrose biosynthesis (energy generation and assimilation) in the source organ (leaf) and the sucrose transportation (energy transportation) to the sink organ (pod) to finally promote yield.

\section{Supplementary Files}


This is a list of supplementary files associated with this preprint. Click to download.

- GmFULa.SUP.final.20210207.docx 